\title{
EQUIVARIANT VECTOR FIELDS ON SPHERES
}

\author{
BY
}

UNNI NAMBOODIRI ${ }^{1}$

\begin{abstract}
We address the following question: If $G$ is a compact Lie group and $S(M)$ is the unit sphere of an $R[G]$-module $M$, then how many orthonormal $G$-invariant vector fields can be found on $S(M)$ ? We call this number the $G$-field number of $M$.

Under reasonable hypotheses on $M$, we reduce this question to determining when the difference of two $G$-vector bundles vanishes in a certain subquotient of the $K O_{G}$-theory of a real projective space. In this general setting, we solve the problem for 2-groups, for odd-order groups, and for abelian groups. If $M$ also has "enough" orbit types (for example, all of them), then we solve the problem for arbitrary finite groups. We also show that under mild hypotheses on $M$, the $G$-field number depends only on the dimensions of the fixed point sets of $M$.
\end{abstract}

ACKNOWLEDGEMENTS. I wish to take this opportunity to express my heartfelt thanks to Peter May for his constant guidance and encouragement, and for his careful reading of this paper; to Jim McClure for many helpful ideas and enlightening conversations and to Mel Rothenberg for being a one-man audience and for all his suggestions and improvements.

Introduction. In 1940 Hopf [14], and later Eckmann [9], observed that a theorem of Hurwitz and Radon could be used to construct orthonormal vector fields on spheres. They showed that if $n=(2 a+1) 2^{c+4 d}$, where $0 \leqslant c \leqslant 3$, then $\rho(n)-1$ orthonormal vector fields can be constructed on $S^{n-1}$, where $\rho(n)=8 d+2^{c}$ is the $n$th Hurwitz-Radon number. Twenty years later, Adams [1] showed that this was best possible; there do not exist $\rho(n)$ orthonormal vector fields on $S^{n-1}$. The purpose of this paper is to address the following question.

If $G$ is a compact Lie group, and $S(M)$ is the unit sphere of a real $G$-module $M$, then how many orthonormal $G$-invariant vector fields (or $G$-fields) can be found on $S(M)$ ?

We shall call this number the $G$-field number of $M$.

Previous work has been done on this problem by Becker [6]. He deals with the case in which $G$ is finite and acts freely on $S(M)$. He shows that, under mild hypotheses, the $G$-field number of $M$ depends only on $G$ and the real dimension of $M$. He also shows that it coincides with the $\Sigma$-field number of $M$ where $\Sigma$ is any 2-Sylow subgroup of $G$.

Received by the editors March 22, 1982.

1980 Mathematics Subject Classification. Primary 57R25, 57S25; Secondary 55R25, 55R50.

${ }^{1}$ The author was tragically killed in an automobile accident on December 23, 1981. The editors thank Peter May and Jim Becker for their help in bringing this paper to publication. Requests for reprints should be addressed to Peter May, University of Chicago. 
Such behaviour will not occur if $G$ does not act freely on $S(M)$, for essentially obvious reasons. Observe that the $G$-field number of a $G$-module $M$ is almost always smaller than its nonequivariant vector field number. The existence of $k G$-fields on $S(M)$ clearly implies the existence of $k$ orthonormal vector fields on each fixed point set, $S\left(M^{H}\right)$. Thus there can be no more than $\min _{H<G}\left\{\rho\left(\operatorname{dim} M^{H}\right)-1\right\} G$-fields on $S(M)$, a number that is smaller, in general, than the nonequivariant vector field number $\rho(\operatorname{dim} M)-1$. In particular, if $G$ has odd order, the $G$-field number of $M$ will generally be smaller than the $\Sigma$-field number. We can also see from the discussion above that the $G$-field number cannot depend only on the dimension of the representation. This, however, raises a natural question.

Does the $G$-field number of $M$ depend only on the dimensions of the fixed point sets of $M$ ?

Surprisingly, the answer is yes-at least for all the cases we treat; if $G$ is finite and $M$ and $N$ are $G$-modules such that $\operatorname{dim} M^{H}=\operatorname{dim} N^{H}$ for each $H<G$, then, under certain hypotheses, the $G$-field numbers of $M$ and $N$ coincide. These hypotheses are probably not necessary. Except for some low-dimensional counterexamples-Sjerve [25] has constructed some if $\operatorname{dim} M=8$-this should be true for all finite groups.

In our calculations, we will, as did Becker, follow the program used to prove the nonequivariant result.

In his proof, Adams relied on the earlier work of Atiyah [3] and James [16] who, using Spanier-Whitehead duality, reduced the vector field problem to a question of whether or not a certain pair of sphere bundles were fibre homotopy equivalent. This, in turn, was equivalent to a certain stunted projective space being coreducible. Adams proceeded to show that it was not, thereby completing his solution.

With some care, the reduction of Atiyah and James can be generalized to the equivariant case. However, we instead make use of a paper by Woodward [29] in which he accomplishes the Atiyah-James reduction without using duality theory. This reduction is simple enough to generalize painlessly to the equivariant case, to show that the $G$-vector field problem is equivalent to the question of whether or not two $G$-sphere bundles are fibre $G$-homotopy equivalent. In particular, we recover Theorem 2.2 of [6].

If $\operatorname{dim} M^{G}$ satisfies some mild conditions, we will show that this problem is in turn equivalent to a stable question. Does the difference of the two $G$-sphere bundles vanish in a certain subquotient of the $K O_{G}$-theory of a real projective space? We then use equivariant homotopy theory to determine when this happens. Since this subquotient is related to $J O_{G}$-theory (in fact is equal to it if $M$ contains all orbit types), we can compute it when $G$ is finite by modifying the techniques of tom Dieck [7] and McClure [21]. Unfortunately, some technical difficulties arise which involve the number of orbit types in $M$. Thus if $G$ is an arbitrary finite group, we solve the problem only if $M$ has "enough" orbit types. This restriction is probably not necessary, as our calculations for abelian groups suggest. We also obtain much sharper results for 2-groups and odd-order groups. Our results on the dependence of the $G$-field number only on the dimensions of the fixed point sets are corollaries of these calculations. 
In order to state our main results, we introduce some terminology. If $n$ is a positive integer written as $(2 a+1) 2^{c+4 d}$, where $a, d \geqslant 0$ and $0 \leqslant c \leqslant 3$, we define three functions $\alpha, \beta$ and $\gamma$ by the following formulas:

$$
\begin{aligned}
& \alpha(n)=8 d+2^{c}-1=\rho(n)-1, \\
& \beta(n)=8 d+2 c+1, \\
& \gamma(n)= \begin{cases}8 d+c+3 & \text { if } c \neq 3 \\
8 d+7 & \text { if } c=3 .\end{cases}
\end{aligned}
$$

We will also employ the function

$$
\operatorname{Dim}(?, G): R O(G) \rightarrow \prod_{H<G} Z
$$

defined by $\operatorname{Dim}(X, G)=\left(\operatorname{dim} X^{H}\right)$.

If $M$ and $V$ are $G$-modules and $V$ is irreducible, we say that the coefficient of $V$ in $M$ is the number of copies of $V$ in $M$. We say that $V$ is of type $R$ if its endomorphism algebra is $R$. We say that $M$ is of type $R$, if it is a sum of type $R$ irreducibles. We define type $C$ and type $H G$-modules similarly.

To state our main results for a 2-group $G$, we consider the action of the Adams operation $\psi^{3}$ on $\operatorname{Irr}(G)$, the set of irreducible $R(G)$-modules. Since 3 is prime to the order of $G, \psi^{3}$ permutes the irreducibles, preserving their types (see Lemma 6.1). Let $\theta_{1}, \ldots, \theta_{r}$ denote the type $R$ orbits, let $\theta_{r+1}, \ldots, \theta_{r+s}$ denote the type $C$ orbits, and let $\theta_{r+s+1}, \ldots, \theta_{r+s+t}$ denote the type $H$ orbits.

TheOREM A. Let $G$ be a 2-group and let $M$ be a G-module. Let $a_{i}$ be the sum of the coefficients in $M$ of all the irreducibles comprising $\theta_{i}$. Let $w_{1}(M), w_{2}(M)$, and $w_{3}(M)$ be the greatest common divisors of $\left\{a_{1}, \ldots, a_{r}\right\},\left\{a_{r+1}, \ldots, a_{r+s}\right\}$, and $\left\{a_{r+s+1}, \ldots, a_{r+s+t}\right\}$, respectively. Define

$$
\delta(M)=\min \left\{\alpha\left(w_{1}(M)\right), \beta\left(w_{2}(M)\right), \gamma\left(w_{3}(M)\right)\right\} .
$$

If $\operatorname{dim} M^{G} \geqslant \max \{3,2(\delta(M)+1)\}$ then the G-field number of $M$ is $\delta(M)$.

THeOREM B. Let $G$ be a 2-group and let $M$ be a G-module such that $\operatorname{dim} M^{G} \geqslant$ $\max \{3,2(\delta(M)+1)\}$. Let $N$ be another $G$-module such that $\operatorname{Dim}(M, G)=$ $\operatorname{Dim}(N, G)$. Then the G-field numbers for $M$ and $N$ coincide.

Our main result for odd-order groups is as follows.

Theorem C. Let $G$ be of odd order and let $M$ be a G-module. Let $\alpha(M)=$ $\min _{H<G}\left\{\alpha\left(\operatorname{dim} M^{H}\right)\right\}$. If $\operatorname{dim} M^{G} \geqslant \max \{3,2(\alpha(M)+1)\}$ then the G-field number of $M$ is $\alpha(M)$.

In order to state our main results for finite groups we require the notion of an "excisive universe".

If $W$ is a $G$-module, let $W^{\infty}$ be the direct sum of infinitely many copies of $W$. If $\left\{W_{i}\right\}$ is a collection of $G$-modules containing the trivial $G$-module, we say that the space $\bigoplus_{i} W_{i}^{\infty}$, topologized as the colimit of its finite-dimensional subspaces, is a universe. 
Given a $G$-module $M$, there is a certain universe-not the obvious one-that can be associated to it, which we call $\mathcal{Q}(M)$. If $\mathscr{Q}(M)$ has "enough" orbit types (for example, all of them), then we say that $\mathcal{Q}(M)$ is an excisive universe. We will make this definition precise in $\S 8$. To streamline our notation, we will write $W H$ for the quotient $N_{G}(H) / H$ when $H<G$.

We are now ready to state Theorems $\mathrm{D}$ and $\mathrm{E}$, our main results for arbitrary finite groups. Theorem D reduces the computation of the $G$-field number of $M$ to computations for certain subquotients of $G$. These subquotients are 2-groups, hence can be dealt with by applying Theorem $\mathrm{A}$. Theorem $\mathrm{E}$ is an immediate consequence of Theorems B and D.

TheOREM D. Let $G$ be a finite group and let $M$ be a G-module such that $\mathscr{Q}(M)$ is an excisive universe. Let $\Phi(2)=\{H<G \mid$ order of $W H$ is odd $\}$, let $H_{2}$ be the smallest normal subgroup of $H$ with 2-primary index, and let

$$
\mu(M)=\min _{H \in \Phi(2)}\left\{H / H_{2} \text {-field number of } M^{H_{2}}\right\} .
$$

If $\operatorname{dim} M^{G} \geqslant \max \{3,2(\mu(M)+1)\}$, then the G-field number of $M$ is $\mu(M)$.

THEOREM E. Let $G$ be finite and let $M$ be a G-module such that $\mathcal{Q}(M)$ is an excisive universe and $\operatorname{dim} M^{G} \geqslant \max \{3,2(\mu(M)+1)\}$. Let $N$ be a G-module such that $\operatorname{Dim}(M, G)=\operatorname{Dim}(N, G)$. Then the G-field numbers of $M$ and $N$ coincide.

Our final result deals with abelian groups and, once again, we need some terminology in order to state it.

If $G$ is abelian, then every irreducible $G$-module $V$ has a unique isotropy subgroup $H$. We say that $V$ is of type I if $\operatorname{dim} V=1$, that $V$ is of type II if $\operatorname{dim} V=2$ and if $Z / 4 Z$ is not a subgroup of $G / H$, and that $V$ is of type III if $\operatorname{dim} V=2$ and $Z / 4 Z$ is a subgroup of $G / H$. Let $\left\{L_{1}, \ldots, L_{r}\right\},\left\{J_{1}, \ldots, J_{s}\right\}$, and $\left\{K_{1}, \ldots, K_{t}\right\}$, respectively, be the sets of isotropy groups of type I, type II, and type III irreducibles. Now suppose given a $G$-module $M$. Then $M$ can be written as a sum $M_{1} \oplus \cdots \oplus M_{r+s+t}$ where each irreducible of $M_{i}$ has isotropy type $L_{i}$ if $1 \leqslant i \leqslant r, J_{i-r}$ if $r+1 \leqslant i \leqslant r+s$, and $K_{i-(r+s)}$ if $r+s+1 \leqslant i \leqslant r+s+t$. Let $b_{i}$ be the number of irreducible summands in a decomposition of $M_{i}$. Then we have the following theorem.

Theorem F. Suppose $G$ is abelian and $M$ is a $G$-module such that $\operatorname{dim} M_{i} \neq 8$ for each i. Let $k_{1}(M)$ and $k_{2}(M)$, respectively, be the greatest common divisors of $\left\{b_{1}, \ldots, b_{r}, 2 b_{r+1}, \ldots, 2 b_{r+s}\right\}$ and $\left\{b_{r+s+1}, \ldots, b_{r+s+t}\right\}$. Then the $G$-field number of $M$ is

$$
\min \left\{\alpha\left(k_{1}(M)\right), \beta\left(k_{2}(M)\right)\right\} .
$$

It is interesting to note the variety of results in these cases. Since $\alpha(n)=\rho(n)-1$, $\alpha(M)=\min _{H<G}\left\{\rho\left(\operatorname{dim} M^{H}\right)-1\right\}$. Thus odd-order groups admit as many $G$-fields as can reasonably be expected. By contrast, 2-groups generally admit a far smaller number.

We will prove these results using somewhat different methods. The proof of Theorem A, the most interesting case, involves tom Dieck's calculations in [7] of the $p$-local $\mathrm{JO}_{G}$-theory for $p$-groups. The proofs of Theorems $\mathrm{C}$ and $\mathrm{D}$ use the techniques 
of McClure [21], who reduces $J O_{G}$ calculations to $p$-local calculations for appropriate $p$-groups. Using representation theory, one can deduce Theorem B from Theorem A. Theorem F can be made to follow from Becker's calculations in [6] and Theorem A once we know from Theorem $\mathrm{D}$ what the answer ought to be.

We remark that our hypotheses on $\operatorname{dim} M^{G}$ are not as onerous as they seem. If $\operatorname{dim} M^{G}>0$, they will be satisfied in all but some low-dimensional cases. In fact, as long as $\operatorname{dim} M^{G}>0$, our results can be used to give a good upper bound on the $G$-field number of $M$. Observe that if $N$ is the direct sum of $t$ copies of $M$ then $S(N)$ is the join of $t$ copies of $S(M)$. Further, any $G$-field $\nu$ on $S(M)$ can be represented by a $G$-map $\nu: S(M) \rightarrow S(M)$ such that $\nu(x) \perp x$ for all $x \in S(M)$. Thus, given $k$ orthonormal $G$-fields on $S(M)$, taking the $t$-fold join of each of them yields $k$ $G$-fields on $S(N)$. Hence the $G$-field number of $M$ is no larger than that of $N$. Now suppose, for example, that $G$ is a 2-group. If $t$ is taken to be large, $N$ will satisfy the hypotheses of Theorem A. Since the functions $\alpha, \beta$, and $\gamma$ depend only on the 2-primary part of their input, the functions $\alpha\left(w_{1}(?)\right), \beta\left(w_{2}(?)\right)$, and $\gamma\left(w_{3}(?)\right)$ will agree on $M$ and $N$ if $t$ is odd. Thus the $G$-field number for $M$ can be no larger than $\delta(M)$. Similar arguments work for Theorems $\mathrm{C}$ and $\mathrm{E}$.

One significant difference between our approach and the classical one is that we do not explicitly construct the required number of $G$-fields on $S(M)$. (In fact, if we were able to do so, we would only require that $\operatorname{dim} M^{G}>0$ in our results.) Generalizations of the standard methods for constructing orthonormal vector fields do not give sufficiently many examples. The simplest such method is to observe that $R^{n}$ is a module over the Clifford algebra $C_{\rho(n)-1}$. Multiplication by the standard algebra generators of $C_{\rho(n)-1}$ produces linear automorphisms of $R^{n}$. Restricting these maps to $S^{n-1}$ gives the vector fields. (See [15, 11.8.1] for details.) In our generalization of this procedure we construct $G$-fields from $G$-automorphisms of $M$. Very often, however, our method yields fewer $G$-fields than the $G$-field number. In any case, since the $G$-field number depends only on the dimensions of the fixed point sets and since the group of $G$-automorphisms of $M$ is more intimately connected with $M$, it seems unreasonable to expect that all the $G$-fields arise from restrictions of $G$-automorphisms. Some of them will probably be nonlinear.

The layout of this paper is as follows. In $\S 1$ we describe the notations and conventions we will use. In $\$ 2$ we prove the $G$-Whitehead and $G$-Freudenthal suspension theorems in suitable generality for our purposes. In $\$ 3$ we reduce the $G$-field problem to a question about $G$-sphere bundles. In $\$ 4$ we prove some general results about desuspending fibre $G$-homotopy equivalences between $G$-sphere bundles. We use them in $\$ 5$ to reduce the general $G$-field problem to a "stable" one. (Our notion of stability is not quite the usual one.) We also introduce the subquotients of $K O_{G}$-theory mentioned earlier and reformulate the preceding results. Throughout the first 5 sections $G$ is to be an arbitrary compact Lie group. In $\S 6$ we prove Theorem $A$ and in $\$ 7$ we prove Theorem B. In $\$ 8$ we introduce the Burnside ring of a general universe and discuss its behaviour relative to that of the Burnside ring $A(G)$. In $\S 9$ we prove Theorem $\mathrm{C}$ and in $\$ 10$ we prove Theorem D. In $\$ 11$ we prove Theorem $\mathrm{F}$ and in $\S 12$ we explicitly construct some $G$-fields on $G$-spheres using the technique of Clifford algebras. 
1. Notations and conventions. In this short section we introduce some notations and state some standing assumptions on our $G$-modules.

Throughout this paper the letters $M, N, V$, and $W$ will denote $G$-modules, $M^{H}$ will denote the $H$-fixed point set of $M, m^{H}$ will denote the dimension of $M^{H}, S(M)$ will denote the unit sphere of the $G$-module $M$, and $S^{M}$ will denote its one point compactification. When dealing with a fixed $G$-space $X$, we will denote the product bundle $X \times M$ by $\mathbf{M}$. If $E$ is a $G$-vector bundle over $X$, then $S(E)$ will denote its unit $G$-sphere bundle, $S^{E}$ will denote its fibrewise one point compactification, and $T E$ will denote its Thom space $S^{E} / X$. If $E$ and $F$ are two $G$-fibre bundles, then we will write $E \sim_{G} F$ when $E$ and $F$ are fibre $G$-homotopy equivalent.

For arguments by induction on the order of $G$, note that we may as well assume that $G$ acts faithfully on $M$. We also assume that $\rho\left(m^{H}\right) \geqslant 2$ if $m^{H} \neq 0$, for if not, there are no $G$-fields on $S(M)$. Many of our theorems deal with equivalent conditions for the existence of $k-1 G$-fields on $S(M)$. We will implicitly assume that $\rho\left(m^{H}\right) \geqslant k$ if $m^{H} \neq 0$.

2. The equivariant Whitehead and Freudenthal suspension theorems. In this section we prove the $G$-Whitehead and $G$-Freudenthal suspension theorems. Versions of the $G$-Whitehead theorem have been proven by other authors: Matumoto in [20] and Waner in [27]. Versions of the $G$-suspension theorem have also been proven by Hauschild in [12] and by Waner in [26]. Their hypotheses, however, are too restrictive for our purposes. We have modified Waner's proofs so that the theorems hold in a more general situation-something that we will need in future sections.

We begin with some definitions. If $X$ is a $G$-space, let $\operatorname{Iso}(X)$ be the set of all the isotropy subgroups of points in $X$. Let $\nu$ be a function from $S$, the set of all subgroups of $G$, to the nonnegative integers. Say that a $G$-map $e: Y \rightarrow Z$ is a $\nu$-equivalence if $e^{H}: Y^{H} \rightarrow Z^{H}$ is a $\nu(H)$-equivalence for all $H$ in $S$. Say that a relative $G$-CW complex $(X, A)$ satisfies $\operatorname{dim}(X, A) \leqslant \nu$ if all the cells of $X$ not in $A$ are of the form $G / H \times D^{n}$ where $n \leqslant \nu(H)$.

We have the following version of $G$-HELP ( $G$-homotopy extension and lifting property). The proof is exactly the same as that given by Waner in $[26,3.3]$ for the special case in which $\nu$ is constant at some $n$.

THEOREM 2.1 (G-HELP). If $(X, A)$ is a relative $G-C W$ complex of dimension $\leqslant \nu$ and $e: Y \rightarrow Z$ is a $\nu$-equivalence and if $f \mid A=h i_{0}$ and $h i_{1}=e g$ in the following diagram, then there exist $\tilde{g}$ and $\tilde{h}$ such that the diagram commutes.

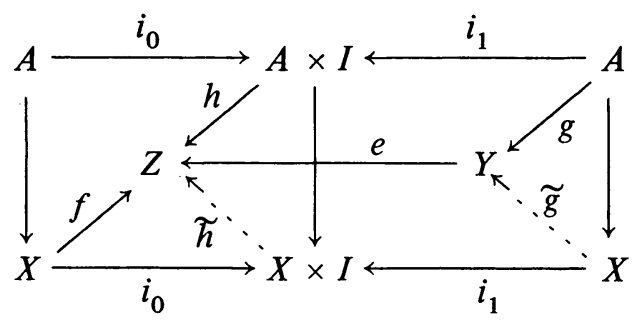


Corollary 2.2 ( $G$-WhiteheAd THEOREM). If $X$ is a $G$-CW complex and $e: Y \rightarrow Z$ is a v-equivalence, then $e_{*}:[X, Y]_{G} \rightarrow[X, Z]_{G}$ is a surjection if $\operatorname{dim} X \leqslant \nu$ and an injection if $\operatorname{dim} X<\nu$.

Proof. The case $(X, \phi)$ gives surjectivity and the case $(X \times I, X \times \partial I)$ gives injectivity.

TheOrem 2.3 ( $G$-Freudenthal SUSPENSION THEOREM). Let $X$ and $Y$ be based $G-C W$ complexes and let $V$ be a G-module. Let $x^{H}=\operatorname{dim} X^{H}$, let $v^{H}=\operatorname{dim} V^{H}$ and let $Y^{H}$ be $y_{H^{-}}$connected. Suppose that $y_{H} \geqslant 1$ whenever $v^{H}>0$. Then

$$
\Sigma^{V}:[X, Y]_{G} \rightarrow\left[\Sigma^{V} X, \Sigma^{V} Y\right]_{G}
$$

is an isomorphism if the following conditions hold:

(i) $x^{H} \leqslant 2 y_{H}$ for every $H \in \operatorname{Iso}(X)$;

(ii) if $K \in \operatorname{Iso}(X)$ with $K<H$ and $v^{K}>v^{H}$, then $x^{H}+1 \leqslant y_{K}$.

The map $\Sigma^{V}$ is an epimorphism if the following conditions hold:

(i) $x^{H} \leqslant 2 y_{H}+1$ for every $H \in \operatorname{Iso}(X)$;

(ii) if $K \in \operatorname{Iso}(X)$ with $K<H$ and $v^{K}>v^{H}$ then $x^{H} \leqslant y_{K}$.

Proof. We will prove the epimorphism case. The proof of the isomorphism case is analogous.

Let $\eta$ and $\varepsilon$ be the unit and counit of the adjoint pair of functors $\left(\Sigma^{V}, \Omega^{V}\right)$. It is well known that the following diagram commutes and that $\varepsilon_{*} \Sigma^{V}$ is an isomorphism.

$$
\begin{array}{ccc}
{[X, Y]_{G}} & \stackrel{\eta_{*}}{\rightarrow} & {\left[X, \Omega^{V} \Sigma^{V} Y\right]_{G}} \\
\downarrow \Sigma^{V} & & \downarrow \Sigma^{V} \\
{\left[\Sigma^{V} X, \Sigma^{V} Y\right]_{G}} & \stackrel{\varepsilon_{*}}{\leftarrow} & {\left[\Sigma^{V} X, \Sigma^{V} \Omega^{V} \Sigma^{V} Y\right]_{G}}
\end{array}
$$

Thus it suffices to show that $\eta_{*}$ is an epimorphism. This, in turn, will follow from the $G$-Whitehead theorem once we show that $\eta^{H}: Y^{H} \rightarrow\left(\Omega^{V} \Sigma^{V} Y\right)^{H}$ is an $x^{H}$-equivalence for every $H$ in Iso $(X)$. To show this we consider the following diagram.

$$
\begin{array}{ccc}
\pi_{r}^{H}(Y)=\left[S^{r}, Y\right]_{H} & \stackrel{\eta_{*}}{\rightarrow} & {\left[S^{r}, \Omega^{V} \Sigma^{V} Y\right]_{H}=\pi_{r}^{H}\left(\Omega^{V} \Sigma^{V} Y\right)} \\
\downarrow \Sigma^{V} & \downarrow \Sigma^{V} \\
{\left[S^{r+V}, \Sigma^{V} Y\right]_{H}} & \stackrel{\varepsilon_{*}}{\leftarrow} & {\left[S^{r+V}, \Sigma^{V} \Omega^{V} \Sigma^{V} Y\right]_{H}}
\end{array}
$$

Since $\varepsilon_{*} \Sigma^{V}$ is again an isomorphism, $\eta^{H}$ will be an $x^{H}$-equivalence if $\Sigma^{V}$ : $\left[S^{r}, Y\right]_{H} \rightarrow\left[S^{r+V}, \Sigma^{V} Y\right]_{H}$ is an isomorphism for $r<x^{H}$ and an epimorphism for $r=x^{H}$. We can reduce the proof of this to obstruction theory by means of the following diagram, in which $R_{1}$ and $R_{2}$ indicate restriction to $H$-fixed point sets.

$$
\begin{aligned}
& {\left[S^{r}, Y\right]_{H} \stackrel{\Sigma^{V}}{\rightarrow} \quad\left[S^{r+V}, \Sigma^{V} Y\right]_{H}} \\
& \simeq \downarrow R_{1} \quad \downarrow R_{2} \\
& {\left[S^{r}, Y^{H}\right] \stackrel{\Sigma^{V^{H}}}{\rightarrow}\left[S^{r+V^{H}}, \Sigma^{V^{H}} Y^{H}\right]}
\end{aligned}
$$


We deduce from the Freudenthal suspension theorem that $\Sigma^{V^{H}}$ is an isomorphism if $r<2 y_{H}+1$ and an epimorphism if $r=2 y_{H}+1$. Since by hypothesis $x^{H} \leqslant 2 y_{H}$ +1 , it suffices to show that $R_{2}$ is injective.

Suppose to the contrary that there exists an $H$-map $g: S^{r+V} \rightarrow \Sigma^{V} Y$ such that $R_{2}(g)=0$. Then $g$ extends to $\tilde{g}$ : $C i \rightarrow \Sigma^{V} Y$ where $i: S^{r+V^{H}} \rightarrow S^{r+V}$ is the inclusion and $C i=S^{r+V} \cup_{i} C S^{r+V^{H}}$ is the cofibre of $i$. We can show that $g=0$ by extending $\tilde{g}$ to $\bar{g}: C S^{r+V} \rightarrow \Sigma^{V} Y$. We compute the obstructions to doing so. Observe that the cells of $C S^{r+V}$ not in $C i$ are of the form $H / K^{+} \wedge D^{n+1}$ where $K<H, v^{K}>v^{H}$, and $n \leqslant r+v^{K}$. Consequently, the obstructions to extending $\tilde{g}$ lie in $\pi_{n}^{K}\left(\Sigma^{V} Y\right)$. Since by hypothesis $x^{H} \leqslant y_{K}$, and since $r \leqslant x^{H}$, we have that $n \leqslant y_{K}+v^{K}$. Since $\left(\Sigma^{V} Y\right)^{K}$ is $y_{K}+v^{K}$ connected, the obstructions vanish. Thus $\tilde{g}$ can be extended to $\bar{g}$ and $R_{2}$ is injective. This completes the proof.

3. Reduction to a question about $G$-sphere bundles. This section is devoted to proving the following theorem.

THeOREM 3.1. Let $G$ be a compact Lie group and let $M$ be a G-module. Let $k$ be a positive integer and let $P(k)$ be $(k-1)$-dimensional real projective space. Let $M \xi$ denote the tensor product of the product $G$-bundle $P(k) \times M$ with the Hopf bundle $\xi$.

If there exist $k-1$ orthonormal $G$-fields on $S(M)$, then $S(M \xi) \sim{ }_{G} S(\mathbf{M})$.

If $S(M \xi) \sim_{G} S(\mathbf{M})$ and for every $H<G$ with $m^{H}>0$ we have that $m^{H} \geqslant 2 k$, then there exist $k-1$ orthonormal $G$-fields on $S(M)$.

The proof of Theorem 3.1 is built up in a sequence of lemmas and proceeds along the same lines as Woodward's proof in the nonequivariant case [29]. In what follows we will omit the proof of a lemma when it is essentially the same as in [29], referring the reader there for details.

Recall that we assume $\rho\left(m^{H}\right) \geqslant \max (2, k)$ if $n^{H} \neq 0$.

LEMMA 3.2. Let $V_{k}(M)$ be the Stiefel manifold of $k$-frames in $M$ with the evident $G$-action. Let $p: V_{k}(M) \rightarrow S(M)$ be the $G$-map given by $p\left(v_{1}, \ldots, v_{k}\right)=v_{1}$. Then there exist $k-1$ orthonormal $G$-fields on $S(M)$ if and only if $p$ admits a G-section $s$.

Let $E_{k}(M)$ denote the space of $Z_{2}$-equivariant maps between $S^{k-1}$ and $S(M)$ in the compact open topology. ( $Z_{2}$ acts by the antipodal map.) $E_{k}(M)$ has a natural $G$-action; given a $Z_{2} \operatorname{map} f: S^{k-1} \rightarrow S(M)$ and $g \in G$ define $g f$ by $(g f)(x)=g(f(x))$. Note that we have a natural map $q: E_{k}(M) \rightarrow S(M)$ defined by $q(f)=f(1)$ where $S^{0}=\{ \pm 1\} \subset S^{k-1}$. Further, $q: E_{1}(M) \rightarrow S(M)$ is obviously a $G$-homeomorphism. There is also a natural $G$-map $i: V_{k}(M) \rightarrow E_{k}(M)$ defined by $i\left(v_{1}, \ldots, v_{k}\right)=f$ where $f\left(\sum a_{i}\right)=\sum a_{i} v_{i}$. Using the definitions of $p, q$, and $i$, we see that the following diagram commutes.

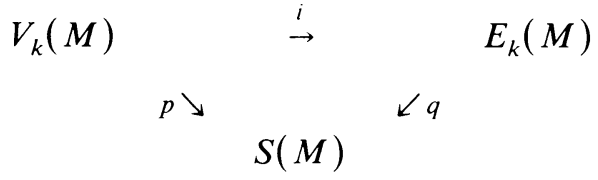

Lemma 3.3. If $p$ admits $a$-section then so does $q$. If $m^{H} \geqslant 2 k$ for every $H<G$ with $m^{i i}>0$, then $a$ G-section of $q$ induces $a$ G-section of $p$. 
Proof. If $\sigma$ is a $G$-section of $p$ then $i \sigma$ is a $G$-section of $q$. The other half of the lemma reduces to an application of the $G$-Whitehead theorem (Corollary 2.2). Let $S$ be the set of all subgroups of $G$. Define a function $\nu$ from $S$ to the nonnegative integers by $\nu(H)=m^{H}-1$. Note that

$$
\left(V_{k}(M)\right)^{H}=V_{k}\left(M^{H}\right) \text { and }\left(E_{k}(M)\right)^{H}=E_{k}\left(M^{H}\right) .
$$

Further, $i: V_{k}\left(R^{N}\right) \rightarrow E_{k}\left(R^{n}\right)$ is a $2 n-2 k-1$ equivalence [11, 1.1]. Thus $i: V_{k}(M)$ $\rightarrow E_{k}(M)$ is a $\nu$-equivalence. Since $\operatorname{dim} S(M) \leqslant \nu$, we can conclude from Corollary 2.2 that a $G$-section of $q$ induces a map $\sigma: S(M) \rightarrow V_{k}(M)$ such that $p \sigma$ is $G$-homotopic to the identity map. Since $p$ is evidently a $G$-fibration, we can $G$-homotope $\sigma$ to an actual section. This completes the proof of 3.3.

If $X$ and $Y$ are $G$-spaces, let $\{X, Y\}_{G}$ be the space of $G$-maps from $X$ to $Y$ in the compact open topology. If $\eta$ and $\xi$ are $G$-fibre bundles over a $G$-space $X$, let $\{\eta, \xi\}_{G}$ be the space of fibrewise $G$-maps from $\eta$ to $\xi$. Our next lemma contains the main part of the proof of Theorem 3.1.

\section{LEMMA 3.4. There exists a homeomorphism}

$$
\Phi:\left\{S(M), E_{k}(M)\right\}_{G} \rightarrow\{S(\mathbf{M}), S(M \xi)\}_{G} .
$$

Say that a $G$-map $f: S(M) \rightarrow S(M)$ is of $G$-degree 1 if it is $G$-homotopic to the identity. Since we can canonically identify the fibres of $S(\mathbf{M})$ and $S(M \xi)$, we can speak of fibrewise $G$-maps between them of fibrewise $G$-degree 1 without ambiguity.

LeMma 3.5. A G-map $s: S(M) \rightarrow E_{k}(M)$ is such that qs has $G$-degree 1 if and only if $\Phi(s): S(\mathbf{M}) \rightarrow S(M \xi)$ has fibrewise $G$-degree 1 .

We are now ready to prove Theorem 3.1.

If there exist $k-1$ orthonormal $G$-fields on $S(M)$, then $E_{k}(M)$ must admit a $G$-section (Lemmas 3.2 and 3.3). But such a section will induce a fibrewise $G$-map of fibrewise $G$-degree 1 (Lemmas 3.4 and 3.5). Applying the equivariant Dold theorem [28, 1.11], we conclude that $S(\mathbf{M}) \sim{ }_{G} S(M \xi)$. Conversely, suppose we have a fibre $G$-homotopy equivalence $f: S(\mathbf{M}) \rightarrow S(M \xi)$. Since $P(k)$ is connected, all the $G$-maps $f_{x}$ are $G$-homotopic. Let $h: S(\mathbf{M}) \rightarrow S(\mathbf{M})$ be a fibrewise $G$-map such that $h_{x}$ is $G$-homotopy inverse to $f_{x}$. Then $f h$ has fibrewise $G$-degree 1 . We can then complete the proof by applying first Lemmas 3.4 and 3.5 and then Lemmas 3.2 and 3.3.

4. Desuspension of fibre $G$-homotopy equivalences. In this section we will prove that if $S(\eta)$ is a $G$-sphere bundle with a fixed point and if $M$ and $N$ are two $G$-modules, then, under certain conditions, a fibre $G$-homotopy equivalence $f$ : $S(\eta \oplus \mathbf{M}) \rightarrow S(\mathbf{N} \oplus \mathbf{M})$ can be desuspended by $M$ to give a fibre $G$-homotopy equivalence $\tilde{f}: S(\eta) \rightarrow S(\mathbf{N})$.

We begin with some terminology. If $S(\eta)$ is a $G$-sphere bundle over $X$, let $D(\eta)$ be the $G$-disk bundle obtained from $S(\eta)$ by taking the fibrewise join of $S(\eta)$ with $X$, thought of as a bundle with trivial fibre. Denote the quotient $D(\eta) / S(\eta)$ by $T \eta$. Of course, if $S(\eta)$ is the unit sphere bundle of a vector bundle $\eta$, then $T \eta$ will be the Thom space of $\eta$. 
LEMMA 4.1. Let $S(\eta)$ be a $G$-sphere bundle over a $G$-CW complex $X$ such that $S(\eta)$ has a fixed point. Let $N$ be a G-module such that

(i) for every $H \in \operatorname{Iso}(X), \operatorname{dim} X^{H} \leqslant n^{H}-2$,

(ii) $n^{G} \geqslant 3$,

(iii) for every $H \in \operatorname{Iso}(S(\eta)), \operatorname{dim} S(\eta)^{H} \leqslant 2 n^{H}-3$.

Then $S(\eta) \sim_{G} S(\mathbf{N})$ if and only if there exists a G-map $f: T \eta \rightarrow S^{N}$ such that for every $x \in X, f_{x}: T \eta_{x} \rightarrow S^{N}$ is a $G_{x}$-homotopy equivalence.

Proof. Suppose $S(\eta) \sim_{G} S(\mathbf{N})$. Then the fibre $G$-homotopy equivalence can be extended radially to induce, upon passage to quotients, a map $T \eta \rightarrow T(\mathbf{N})$. Composing this map with the projection $p: T(\mathbf{N}) \rightarrow S^{N}$, we obtain a map $f$ with the desired properties.

Conversely, consider the cofibre sequence for the inclusion $S(\eta) \rightarrow D(\eta)$. Application of the functor $\left[?, S^{N}\right]_{G}$ produces the following exact sequence:

$$
\cdots \rightarrow\left[\Sigma S(\eta), S^{N}\right]_{G} \stackrel{\phi}{\rightarrow}\left[T \eta, S^{N}\right]_{G} \rightarrow\left[D \eta, S^{N}\right]_{G} \rightarrow \cdots .
$$

Since $D \eta$ is $G$-homotopy equivalent to $X$ and since $\operatorname{dim} X^{H} \leqslant n_{H}-2$, we can show using obstruction theory that $\left[D \eta, S^{N}\right]_{G}=0$. Consequently $\phi$ is onto. Let $\tilde{f}$ be in the preimage of $f$ under $\phi$. It is easy to see that $\tilde{f}_{x}: \Sigma S\left(\xi_{x}\right) \rightarrow S^{N}$ will still be a $G_{x}$-homotopy equivalence.

Observe that our hypotheses on $N$ allow us to invoke Theorem 2.3 to conclude that the suspension map

$$
\Sigma:[S(\eta), S(N)]_{G} \rightarrow\left[\Sigma S(\eta), S^{N}\right]_{G}
$$

is onto; so let $\bar{f}$ be in the preimage of $\tilde{f}$. Then for every $x \in X$ and for every $H<G_{x}$, $\operatorname{deg} \bar{f}_{x}^{H}=\operatorname{deg} \tilde{f}_{x}^{H}$. Thus the fibrewise $G$-map $f^{\prime}: S(\eta) \rightarrow S(\mathbf{N})$ induced by $\bar{f}$ is such that $f_{x}^{\prime}: S\left(\eta_{x}\right) \rightarrow S(N)$ is a $G_{x}$-homotopy equivalence. Hence, by the equivariant Dold theorem [28, 1.11], $f^{\prime}$ is a fibre $G$-homotopy equivalence.

We are now ready to prove the main theorem of this section.

THEOREM 4.2. Let $S(\eta)$ be a G-sphere bundle with G-fixed point over a G-CW complex $X$ and let $N$ be a G-module. Assume the following conditions:

(1) For every $H \in \operatorname{Iso}(X), \operatorname{dim} X^{H} \leqslant n^{H}-2$.

(2) $n^{G} \geqslant 3$.

(3) For every $K \in \operatorname{Iso}(S(\eta)), \operatorname{dim} S(\eta)^{K} \leqslant 2 n^{K}-3$.

(4) For every $H \in \operatorname{Iso}(T \eta), \operatorname{dim} T \eta^{H} \leqslant 2 n^{H}-1$.

Let $V$ be a G-module such that if $H$ and $K$ are in $\operatorname{Iso}(T \eta)$ with $K<H$ and $v^{K}>v^{H}$, then $\operatorname{dim} T \eta^{H} \leqslant n^{K}-1$. Then $S(\eta) \sim_{G} S(\mathbf{N})$ if and only if $S(\eta \oplus \mathbf{V}) \sim_{G} S(\mathbf{N} \oplus \mathbf{V})$.

Proof. Evidently we need only prove the converse. This is immediate, for we have cunningly chosen our hypotheses so that $S(\eta)$ and $S(\eta \oplus \mathbf{V})$ both satisfy the hypotheses of Lemma 4.1. To complete the proof then, it is enough to construct a $G$-map $f: T \eta \rightarrow S^{N}$ such that for every $x \in X, f_{x}: T \eta_{x} \rightarrow S^{N}$ is a $G_{x}$-homotopy equivalence. We can do so since the map

$$
\Sigma^{V}:\left[T \eta, S^{N}\right]_{G} \rightarrow\left[\Sigma^{V} T \eta, S^{V+N}\right]_{G}
$$

is onto by Theorem 2.4 . 
5. Reduction of the general case to the stable case. In $\S 3$ we reduced the $G$-vector field problem to a question of whether or not two $G$-sphere bundles were fibre $G$-homotopy equivalent. In this section we use Theorem 4.2 to show that this is equivalent to the two bundles being "stably" fibre $G$-homotopy equivalent.

We begin by applying Theorem 4.2 to the $G$-sphere bundle $S(M \xi)$ over $P(k)$. We have the following corollary.

COROLlaRY 5.1. Let $V$ be a $G$-module such that

(i) for every $H$ and $K$ in $\operatorname{Iso}(M)$ with $K<H$ and $v^{K}>v^{H}$ we have that $m^{K}>m^{H}$.

(ii) $S(M \xi \oplus \mathbf{V}) \sim_{G} S(\mathbf{M} \oplus \mathbf{V})$.

Suppose $m^{G} \geqslant \max \{3, k+1\}$. Then $S(M \xi) \sim_{G} S(\mathbf{M})$.

Proof. We will apply Theorem 4.2. To check its hypotheses, we must verify the following condition:

(iii) If $K<H$ and $v^{K}>v^{H}$ then $m^{K}-m^{H} \geqslant k$.

We will show that (i) and (ii) imply (iii). Upon restriction to $H$-fixed point sets, (ii) implies that $m^{H}(\xi-1)=0$ in $\tilde{J} O(P(k))$. This group is cyclic of order $a_{k}$ with generator $\xi-1$, and $a_{k} \geqslant k$ (see e.g. [15] for a table of values for $a_{k}$ ). Thus $a_{k}$ divides $m^{H}$. Similarly, $a_{k}$ divides $m^{K}$. Therefore $a_{k}$ divides $m^{K}-m^{H}$ and so, if $m^{K}-m^{H}>0$, then $m^{K}-m^{H} \geqslant k$.

Let $\operatorname{Gap}(M)=\left\{G\right.$-modules $V \mid$ if $K<H$ and $v^{K}>v^{H}$ then $\left.m^{K}>m^{H}\right\}$. Combining 5.1 and 5.2 we see that under mild hypotheses on $M, S(M \xi)$ and $S(\mathbf{M})$ are fibre $G$-homotopy equivalent if and only if they become fibre $G$-homotopy eqivalent when stabilized by some $V \in \operatorname{Gap}(M)$.

$\operatorname{Gap}(M)$ is closed under direct sum and contains the trivial $G$-module. We define the $G$-universe $\mathcal{Q}(M)$ promised in the introduction by

$$
\text { थ }(M)=\underset{W_{i} \in \operatorname{Gap}(M) \cap \operatorname{Irr}(G)}{W_{i}^{\infty} .}
$$

We shall often suppress the $M$ and write $\mathcal{Q}$ instead when the module $M$ is understood. If $H<G$ then $\mathscr{U}(M)$ thought of as an $H$-space will be an $H$-universe. When we want $H$ to be the ambient group rather than $G$, we will write $\mathcal{Q}(M, H)$ or थ( $H)$.

Suppose $X$ is a $G$-space such that $\operatorname{Iso}(X) \subset \operatorname{Iso}(थ)$. Then we can define the following subgroup of $K O_{G}(X)$ :

$$
K O_{G}(X ; \text { U })=\left\{E-F \in K O_{G}(X) \mid E_{x}, F_{x} \subset \mathcal{Q}\left(M, G_{x}\right) \text { for each } x \in X\right\} .
$$

(Here the inclusion is understood as inclusion up to isomorphism.) Let

$$
\begin{array}{r}
T O_{G}(X ; \text { थ })=\left\{E-F \in K O_{G}(X ; \text { थ }) \mid S(E \oplus \mathbf{V}) \sim_{G} S(F \oplus \mathbf{V})\right. \\
\text { for some } V \in \operatorname{Gap}(M)\},
\end{array}
$$

and define $J O_{G}(X ; \mathcal{Q})$ to be the quotient $K O_{G}(X ; \mathcal{Q}) / J O_{G}(X ;$ थ). We can now combine our preceding results in the following theorem.

THEOREM 5.2 If there exist $k-1 G$-fields on $S(M)$, then $M \xi-\mathbf{M}$ vanishes in $J O_{G}(P(k))$.

If $m^{G} \geqslant \max \{3,2 k\}$ and if $M \xi-\mathbf{M}$ vanishes in $J O_{G}(P(k)$; Q $)$, then there exist $k-1 G$-fields on $S(M)$. 
6. The proof of Theorem A. Before proceeding to the proof, we record some elementary general facts about Adams operations on $R O(G)$.

LEMMA 6.1. Let $V$ be a real $G$-module and let $k$ be prime to the order of $G$.

(i) If $V$ is irreducible, then so is $\psi^{k}(V)$.

(ii) $\psi^{k}(V)$ has the same type as $V$.

(iii) If $H<G$, then $\operatorname{dim} V^{H}=\operatorname{dim} \psi^{k}(V)^{H}$.

Proof. We first note that $\psi^{k}(V)$, which is given a priori as a difference class, is in fact represented by a $G$-module when $k$ is prime to the order of $G$. This is clear if $V$ is the image of a complex $G$-module $V^{\prime}$ in view of the explicit description of $\psi^{k}\left(V^{\prime}\right)$ given in [30,3.1]. Thus $2 \psi^{k}(V)$ is represented by a $G$-module for any $V$, and it follows that the same is true for $\psi^{k}(V)$.

(i) By periodicity $[30,3.2], V=\psi^{s} \psi^{k}(V)$ for some positive integer $s$ prime to the order of $G$. From the remarks above, we see that a nontrivial decomposition of $\psi^{k}(V)$ would yield a nontrivial decomposition of $V$.

(ii) An irreducible $G$-module $V$ has type $R, C$ or $H$ depending on whether $\operatorname{dim}\left(\operatorname{Hom}_{G}(V, V)\right)$ is 1,2 or 4 . From elementary character theory,

$$
\operatorname{dim}\left(\operatorname{Hom}_{G}(V, V)\right)=\frac{1}{|G|} \sum_{g \in G} \chi(g, V)^{2} .
$$

Since $\chi\left(g, \psi^{k}(V)\right)=\chi\left(g^{k}, V\right)$ and the map $g \rightarrow g^{k}$ permutes the elements of $G$, we calculate that

$$
\operatorname{dim} \operatorname{Hom}_{G}(V, V)=\operatorname{dim} \operatorname{Hom}_{G}\left(\psi^{k}(V), \psi^{k}(V)\right) .
$$

(iii) We have

$$
\operatorname{dim}\left(V^{H}\right)=\frac{1}{|H|} \sum_{h \in H} \chi(h, V) .
$$

Again, we calculate using this formula that $\operatorname{dim}\left(V^{H}\right)=\operatorname{dim}\left(\psi^{k}(V)^{H}\right)$.

In the rest of this section $G$ will denote a 2-group. We will deduce Theorem $\mathrm{A}$ from Theorem 6.2 below. In order to state it, however, we need some more terminology.

Suppose $E$ and $F$ are vector bundles in $K O_{G}(X ; \mathcal{Q})$. Then, following [7, §11.1], we say that $E \sim_{\text {loc }} F$ if, for each $H<G$, there exists a $G$-module $V(H) \subset \mathscr{U}$ and $G$-maps

$$
f: S(E \oplus \mathbf{V}(\mathbf{H})) \rightarrow S(F \oplus \mathbf{V}(\mathbf{H})) \quad \text { and } \quad g: S(F \oplus \mathbf{V}(\mathbf{H})) \rightarrow S(E \oplus \mathbf{V}(\mathbf{H}))
$$

such that $f_{x}^{H}$ and $g_{x}^{H}$ are homotopy equivalences for each $x \in X$. Let

$$
T O_{G}^{\text {loc }}(X ; \text { थ })=\left\{E-F \in K O_{G}(X ; \text { थ }) \mid E \sim_{\text {loc }} F\right\}
$$

and let $J O_{G}^{\text {loc }}(X ;$ Q $)$ be the quotient $K O_{G}(X ;$ थ $) / T O_{G}^{\text {loc }}(X ;$ Q $)$. Note that $T O_{G}(X ;$ थ $) \subset T O_{G}^{\text {loc }}(X$; थ $)$ so that $J O_{G}^{\text {loc }}(X ;$ थ $)$ is a quotient of $J O_{G}(X$; थ $)$. Note also that Lemma 6.1(iii) implies

$$
\psi^{3}\left(K O_{G}(X ; \mathscr{Q})\right) \subset K O_{G}(X ; \mathscr{Q}) .
$$


THEOREM 6.2. The following sequence is exact, where $J$ is the quotient map:

$$
K O_{G}(P(k) ; \mathscr{Q})_{(2)}^{\stackrel{1-\psi^{3}}{\rightarrow}} K O_{G}(P(k) ; \mathscr{Q})_{(2)} \stackrel{J}{\rightarrow} J O_{G}^{\operatorname{loc}}(P(k) ; \mathscr{U})_{(2)} .
$$

Tom Dieck [7, 11.4.1] has proven this result for all compact $G$-spaces if $\mathscr{Q}$ is $G R^{\infty}$, the universe generated by the regular representation. We modify tom Dieck's proof to work in our situation.

We remark that $\operatorname{Ker} J \subset \operatorname{Im}\left(1-\psi^{3}\right)$, for this is true when $\mathscr{Q}$ is $G R^{\infty}$. It remains to show the other containment. Some of tom Dieck's proof will work without modification. However, since we can stabilize only by submodules of $\mathscr{Q}$, there are some problems. We need the following proposition.

Proposition 6.3. Let $E$ be a $G$-vector bundle on $P(k)$. Then there exists a fibrewise $G$-map $f: S(E) \rightarrow S\left(\psi^{3}(E)\right)$ such that $f_{x}$ has degree a power of 3 for each $x \in P(k)$.

This proposition is an immediate consequence of the following lemma whose lengthy proof we defer to the end of this section.

LEMMA 6.4. Let $M$ be a G-module. Let $Z_{2}$ act antipodally on $S(M)$ and $S\left(\psi^{3}(M)\right)$. Then there exists a $G \times Z_{2}$-map $f: S(M) \rightarrow S\left(\psi^{3}(M)\right)$ whose degree is a power of 3 .

Proof of Proposition 6.3. Observe that any $G$-vector bundle on $P(k)$ is either a product bundle, or of the form $\left[S^{k-1} \times M\right] / Z_{2}$ for some $G$-module $M$ [1]. Further, if $E$ is a $G$-vector bundle of this latter type, then $\psi^{3}(E)$ is of the form $\left[S^{k-1} \times \psi^{3}(M)\right] / Z_{2}$. Thus the $G \times Z_{2}$-map $M \rightarrow \psi^{3} M$ of Lemma 6.3 will yield the desired map $f$ in either case; in the product bundle case this is immediate, and in the other case this follows upon passage to quotients.

If $E$ and $F$ are vector bundles in $K O_{G}\left(X\right.$; थ), we say that $E \sim_{p} F$ if there exist $V \subset \mathscr{Q}$ and fibrewise $G$-maps $f: S(E \oplus \mathbf{V}) \rightarrow S(F \oplus \mathbf{V})$ and $g: S(F \oplus \mathbf{V}) \rightarrow S(E \oplus \mathbf{V})$ such that $f_{x}$ and $g_{x}$ have degree prime to $p$ for each $x \in X$. Let

$$
T O_{G}(X ; \text { थ } ; p)=\left\{E-F \in K O_{G}(X ; \text { थ }) \mid E \sim_{p} F\right\}
$$

and let $J O_{G}(X ; \mathcal{Q} ; p)$ be the quotient $K O_{G}(X ; \mathcal{Q}) / T O_{G}(X ; \mathcal{Q} ; p)$. Then we have the following lemma.

LEMMA 6.5. The canonical quotient map

$$
J O_{G}^{\text {loc }}(P(k) ; \text { थ })_{(2)} \rightarrow J O_{G}(P(k) ; \text { थ } ; 2)_{(2)}
$$

is an isomorphism.

Proof. The proof is exactly the same as that of $[7,11.4 .2]$. We need only check that $1 \in \operatorname{ISO}(\mathcal{Q})$ so that we can use the transfer $\operatorname{ind}_{1}^{G}$ in stable $G$-cohomotopy. This follows from our assumptions on $M$.

We can complete the proof of Theorem 6.1 with the following lemma.

Lemma 6.6. $\left(1-\psi^{3}\right) K O_{G}(P(k) \text {; } \mathscr{U})_{(2)} \subset T O_{G}(P(k) \text {; } Q ; 2)_{(2)}$.

Proof. The proof is exactly the same as that of $[7,11.4 .3]$, with the necessary input in our case being provided by Proposition 6.3 and Lemma 6.5. 
With Theorem 6.2 on hand, we return to the proof of Theorem A. Note that if $K$ is a $p$-group, $M$ is a $K$-module, and $f: S(M) \rightarrow S(M)$ is a $K$-map, then $\operatorname{deg} f^{H} \equiv$ $\operatorname{deg} f(p)$ for each $H<K$. This can easily be seen by considering the isomorphism between the Burnside ring and the 0th stable cohomotopy group [7, 8.5.1]. Thus, if $M \xi-\mathbf{M}$ vanishes in $J O_{G}^{\text {loc }}(P(k)$; $Q)$, then there exists a $G$-module $V \subset \mathscr{U}$ and a fibrewise $G$-map $f: S(M \xi \oplus \mathbf{V}) \rightarrow S(\mathbf{M} \oplus \mathbf{V})$ such that $\operatorname{deg} f_{x}^{H}$ is prime to $p$ for each $x \in P(k)$. Thus by the equivariant Dold theorem mod $k[13,0.2]$, there is an integer $t$, prime to $p$, such that $S(t(M \xi \oplus \mathbf{V})) \sim_{G} S(t(\mathbf{M} \oplus \mathbf{V}))$. Hence $M \xi-\mathbf{M}$ vanishes in $J O_{G}(P(k) ; \mathcal{Q})_{(p)}$. Since $M \xi-\mathbf{M}$ has 2-primary order, it vanishes in $J O_{G}(P(k) \text {; थ })_{(2)}$ if and only if it vanishes in $J O_{G}(P(k)$; $\mathscr{Q})$. Consequently the proof of Theorem A reduces to the algebraic question:

$$
\text { When is } M \xi-\mathbf{M} \text { in the image of } 1-\psi^{3} \text { ? }
$$

To investigate this question we need some more terminology. Let $R(R ; \mathcal{Q})$ be the Grothendieck group of type $R G$-modules in $\operatorname{Gap}(M)$. Define $R(C ;$ Q $)$ and $R(H ; \mathcal{Q})$ similarly and let $R O(G ; \mathscr{Q})$ be the direct sum of these groups. Then we can deduce from [23] that

$$
\begin{aligned}
K O_{G}(P(k) ; \text { थ })= & R O(G ; \text { U }) \oplus[\tilde{K} O(P(k)) \otimes R(R ; \text { ひ })] \\
& \oplus[\tilde{K} U(P(k)) \otimes R(C ; \text { थ })] \oplus[\tilde{K} S p(P(k)) \otimes R(H ; \text { U })]
\end{aligned}
$$

and, as mentioned above, each of these summands will be invariant under the action of $1-\psi^{3}$. Further, since $\tilde{K} O(P(k)), \tilde{K} U(P(k))$, and $\tilde{K} S p(P(k))$ are cyclic 2-groups, localizing $K O_{G}(P(k) ; \mathscr{Q})$ at 2 amounts to localizing the first summand, $R O(G ;$ थ $)$, at 2 .

Keeping this decomposition in mind, it is easy to see that if we write $M$ as $M_{1} \oplus M_{2} \oplus M_{3}$ where $M_{1}$ is of type $R, M_{2}$ is of type $C$, and $M_{3}$ is of type $H$, then $M \xi-\mathbf{M}$ is in the image of $1-\psi^{3}$ if and only if there exist (possibly virtual) $G$-modules $N_{1}, N_{2}$, and $N_{3}$ of type $R, C$, and $H$, respectively, such that $\left(1-\psi^{3}\right) N_{i} \equiv$ $M_{i}$ modulo the order of the $K$-group involved. Recall the notations introduced above Theorem A.

LEMMA 6.7. Let $N$ be a $G$-module of type $R$. Write

$$
N=\sum_{j} a_{1, j} \dot{V}_{1, j}+\cdots+\sum_{j} a_{r, j} V_{r, j}
$$

where $\left\{V_{i, j}\right\}$ for fixed is the set of irreducibles in the orbit $\theta_{i}$.

Then there exists a (possibly virtual) G-module $W$ of type $R$ such that $\left(1-\psi^{3}\right) W \equiv$ $N\left(2^{t}\right)$ if and only if $\sum_{j} a_{i, j} \equiv 0\left(2^{t}\right)$ for $1 \leqslant i \leqslant r$. Further, the analogous statements for type $C$ and type $H$ modules also hold.

Proof. If $\left(1-\psi^{3}\right) W \equiv N$ then, for every $i \leqslant r$, there is a $W_{i}$ such that $\left(1-\psi^{3}\right) W_{i}$ $\equiv \Sigma_{j} a_{i, j} V_{i, j}\left(2^{t}\right)$. Hence it is enough to consider $N=\Sigma_{j} a_{j} V_{i, j}$ for a fixed $i$. Let $s$ be the number of irreducibles in $\vartheta_{i}$. Write $W_{i}=\Sigma_{j} c_{j} V_{i, j}$ and order the $V_{i, j}$ so that $\psi^{3} V_{i, j}=V_{i, j+1}$ if $j<s$ and $\psi^{3} V_{i, s}=V_{i, 1}$. Then

$$
\left(1-\psi^{3}\right) W_{i}=\left(c_{1}-c_{s}\right) V_{i, 1}+\left(c_{2}-c_{1}\right) V_{i, 2}+\cdots+\left(c_{s}-c_{s-1}\right) V_{i, s} .
$$

If $\left(1-\psi^{3}\right) W_{i}=N_{i}$, then $a_{i, j} \equiv\left(c_{j}-c_{j-1}\right)\left(2^{t}\right)$ if $j \neq 1$ and $a_{i, 1} \equiv\left(c_{1}-c_{s}\right)\left(2^{t}\right)$. 
Thus

$$
\sum_{j} a_{i, j} \equiv\left(c_{1}-c_{s}\right)+\sum_{2 \leqslant j \leqslant s}\left(c_{j}-c_{j-1}\right)
$$

Since the sum on the right is clearly zero, we deduce one half of the lemma.

Conversely, if $\Sigma_{j} a_{i, j} \equiv 0\left(2^{t}\right)$, then we can define a $G$-module $W_{i}$ by $W_{i}=$ $\sum_{1 \leqslant j \leqslant s} c_{j} V_{i, j}$, where $c_{j}=\sum_{1 \leqslant q \leqslant j} a_{i, q}$. A quick check shows that, indeed, $\left(1-\psi^{3}\right) W_{i} \equiv$ $\sum_{j} a_{i, j} V_{i, j}\left(2^{t}\right)$. This completes the proof.

We are now ready to prove Theorem $A$.

Proof of Theorem A. Using Lemma 6.7 and the calculations in [1] of the orders of the various $K$-theories of $P(k)$, we can now decide exactly when $M \xi-\mathbf{M}$ is in the image of $1-\psi^{3}$. Clearly $\alpha\left(w_{1}(M)\right)+1$ is the largest $k$ for which $M_{1} \xi-\mathbf{M}_{1}$ is in the image of $\left(1-\psi^{3}\right)$, hence is also the largest $k$ for which $M_{1} \xi-\mathbf{M}_{1}$ vanishes in $J O_{G}(P(k) \text {; थ })_{(2)}$. The same is true for $\beta\left(w_{2}(M)\right)+1$ and $M_{2} \xi-\mathbf{M}_{2}$ and for $\gamma\left(w_{3}(M)\right)+1$ and $M_{3} \xi-\mathbf{M}_{3}$. Thus if $m^{G} \geqslant \max \{3,2(\delta(M)+1)\}$, then we deduce from Theorem 5.3 that there are $\delta(M) G$-fields on $S(M)$. In view of Theorem 3.1 and the preceding calculations, there are no more. This completes the proof.

We conclude this section with the promised proof of Lemma 6.4.

Proof of Lemma 6.4. Clearly it is enough to prove the lemma for an irreducible $G$-module $V$; we can then obtain $f$ by taking the join of the resulting maps. The lemma is immediate if $\operatorname{dim}_{R} V=1$, for then $\psi^{3}(V)=V$. If $\operatorname{dim}_{R} V=2$, then the $O(2) \times Z_{2}$-map $\tilde{f}: S(V) \rightarrow S\left(\psi^{3}(V)\right)$ given by $\tilde{f}(z)=z^{3}$ will induce $f$. (See [2,p. 193].) If $H<G$ and $V$ is induced up from an irreducible $H$-module $W$ which admits an $H \times Z_{2}$-map $\phi: S(W) \rightarrow S\left(\psi^{3}(W)\right)$ of 3-primary degree, then the $G \times Z_{2}$-map $\operatorname{ind}_{H}^{G}(\phi): S(V) \rightarrow S\left(\operatorname{ind}_{H}^{G}\left(\psi^{3}(V)\right)\right.$ will also have 3-primary degree. Since $G$ is a 2-group, 3 is prime to [G;H]. Thus $\operatorname{ind}_{H}^{G} \psi^{3}(W) \cong \psi^{3}\left(\operatorname{ind}_{H}^{G} W\right)$. (See [22, p. 70].) Composing $\operatorname{ind}_{H}^{G}(\phi)$ with this isomorphism, we obtain the desired map $f$.

Combining these observations, we see that it is enough to show that all irreducible $R[G]$-modules can be induced up from 1- and 2-dimensional modules over subgroups of $G$. (Note that this is a refinement of $[22,2.4]$ for the case of 2-groups.) By means of the following lemma due to G. Glauberman, we can reduce this problem to the case of cyclic, generalized quaternion, dihedral, or semidihedral groups - that is, all 2-groups which do not have a noncyclic abelian normal subgroup of order 4 $[10,4.4]$.

LEMMA 6.8. Let $M$ be a faithful irreducible G-module and suppose that $G$ has a normal noncyclic subgroup $H$ of order 4. Let $C(H)$ denote the centralizer of $H$ in $G$. Then there exists an irreducible $C(H)$-module $V$ such that $M=\operatorname{ind}_{C(H)}^{G} V$.

Proof. Since $G$ admits a faithful irreducible $G$-module, its center, $Z$, must be cyclic; for $M^{K}$ will be a submodule of $M$ for any $K<Z$. Consequently $H$ is not contained in $Z$. Since any normal subgroup of a p-group intersects its center nontrivially, we can present $H$ in the following manner:

$$
H=\left\langle h, k \mid h^{2}=k^{2}=1 ; h, k \notin Z ; h k=k h \in Z\right\rangle .
$$


It is easy to see that $M=M^{h} \oplus M^{k}$ as vector spaces and that if $g \in G$ and $\lambda_{g}$ : $M \rightarrow M$ is the $g$-action map, then one of two things must occur. Either $\lambda_{g}\left(M^{h}\right)=M^{h}$ and $\lambda_{g}\left(M^{k}\right)=M^{k}$-in which case $g \in C(H)$-or $\lambda_{g}\left(M^{h}\right)=M^{k}$ and $\lambda_{g}\left(M^{k}\right)=$ $M^{h}$-in which case $g \notin C(H)$. Thus we can conclude from $[24,19]$ that $M=$ $\operatorname{ind}_{C(H)}^{G} M^{h}$.

Since the center of $C(H)$ is not cyclic, $C(H)$ has no faithful irreducible representations. Passing to quotients and iterating the argument as necessary, we reduce to the case of a group $G$ which contains no noncyclic normal abelian subgroup of order 4. Such groups, as we noted above, must be of one of four well understood types.

Since cyclic and dihedral groups only have irreducibles of dimension 1 or 2, we may assume that $G$ is generalized quaternion or semidihedral. Since both have a normal cyclic subgroup $H$ of index 2, it follows from [24, Prop. 24] that all irreducible $C[G]$-modules $V$ are such that $\operatorname{dim}_{C} V=1$ or 2 . We may assume that $\operatorname{dim}_{C} V=2$ and that $\operatorname{End}_{R[G]}(V)=C$ or $H$. (If $\operatorname{End}_{R[G]}(V)$ were $R$, then $V \otimes_{R} C$ would be an irreducible $C[G]$-module of complex dimension 4.) Then there exists an irreducible $R[H]$-module $W$ such that $\operatorname{dim}_{R} W=2$ and $\operatorname{ind}_{H}^{G} W=V[\mathbf{2 4}$, Theorem 16]. Thus we are reduced to the case of an irreducible of dimension 2. The lemma now follows from our previous remarks.

7. The proof of Theorem B. Let $G$ be a 2-group, let $M$ be a $G$-module such that $m^{G} \geqslant \max \{3,2(\delta(M)+1)\}$, and let $N$ be a $G$-module such that $\operatorname{Dim}(M, G)=$ $\operatorname{Dim}(N, G)$. We have calculated the $G$-field number of $M$, and we must show that $N$ has the same $G$-field number. Clearly it suffices to show that the functions $w_{1}, w_{2}$, and $w_{3}$ coincide on $M$ and $N$. We will deduce this from the following two theorems, the first of which is of independent interest.

TheOrem 7.1. Suppose $G$ is a 2-group and $M$ and $N$ are irreducible $R[G]$-modules such that $\operatorname{Dim}(M, G)=\operatorname{Dim}(N, G)$. Then there is a positive integer $r$ such that $\psi^{3^{r} M}=N$.

In order to state the next theorem we need some terminology. Let $\sim$ be the equivalence relation on $\operatorname{Irr}(G)$ specified by $V \sim W$ if and only if $\operatorname{Dim}(V, G)=$ $\operatorname{Dim}(W, G)$. Let $\bigodot_{1}, \ldots, \bigodot_{p}$ be the equivalence classes of irreducibles under this relation.

THEOREM 7.2. Let $G$ be a finite group and let $M$ and $N$ be G-modules such that $\operatorname{Dim}(M, G)=\operatorname{Dim}(N, G)$. Let $m_{i}$ and $n_{i}$ be the sum of the coefficients in $M$ and $N$, respectively, of the irreducibles in $\mathcal{C}_{i}$. Then $m_{i}=n_{i}$ for each $i$.

Theorem B is an immediate consequence of these two theorems. From Theorem 7.1 we deduce that an equivalence class of irreducibles actually corresponds to an orbit under the action of $\psi^{3}$. Since the integers $w_{1}(M), w_{2}(M)$, and $w_{3}(M)$ depend only on the sum of the coefficients in $M$ of the irreducibles in the various orbits, they must concur with $w_{1}(N), w_{2}(N)$, and $w_{3}(N)$, respectively.

We will spend the remainder of this section proving Theorems 7.1 and 7.2.

To prove 7.1, we may assume that $M$ and $N$ are faithful; if not, since $\operatorname{Dim}(M, G)$ $=\operatorname{Dim}(N, G)$, we see that the largest subgroup $K$ of $G$ that fixes $M$ must be the 
largest subgroup of $G$ fixing $N$. Thus $M$ and $N$ are $G / K$-modules and again $\operatorname{Dim}(M, G / K)=\operatorname{Dim}(N, G / K)$.

We need the following observation, which we record as a lemma for future reference.

Lemma 7.3. If $J<H \triangleleft G$ and $V$ and $W$ are $H$-modules, then $\operatorname{dim}\left(\operatorname{ind}_{H}^{G} V\right)^{J}=$ $\operatorname{dim}\left(\operatorname{ind}_{H}^{G} W\right)^{J}$ if and only if $\operatorname{dim} V^{J}=\operatorname{dim} W^{J}$.

Thus it will be enough to show that all $G$-modules can be induced up from modules over some subgroup for which Theorem 7.1 holds. This is quite straightforward if $G$ does not have a normal noncyclic subgroup of order 4 . The remaining cases can be reduced to this case by means of Glauberman's lemma (Lemma 6.8). Suppose $G$ admits a noncyclic normal subgroup $H$ of order 4 . Then $M=\operatorname{ind}_{C(H)}^{G} V$ and $N=\operatorname{ind}_{C(H)}^{G} W$, where $C(H)$ is the centralizer of $H$ and $V$ and $W$ are $C(H)$-modules. Moreover, since $C(H)$ is clearly normal in $G$, we deduce from Lemma 7.3 that $\operatorname{Dim}(V, C(H))=\operatorname{Dim}(W, C(H))$. Since the center of $C(H)$ is not cyclic, $C(H)$ has no faithful irreducible representations. Passing to quotients, and iterating the argument as necessary, we reduce to the case of a group $G$ which contains no normal noncyclic subgroup of order 4 . Recall again that these are precisely the cyclic, generalized quaternion, dihedral, and semidihedral groups $[10,4.4]$. We will first prove the cyclic case and then reduce the remaining cases to the cyclic case.

LEMMA 7.4. All inequivalent free irreducible $R\left[Z / 2^{m} Z\right]$-modules are permuted in a single orbit by $\psi^{3}$.

Proof. This is immediate for $Z / 2 Z$ and $Z / 4 Z$ as they both have a unique such representation. So we may assume $m \geqslant 2$.

Let $R$ denote the ring $Z / 2^{m} Z$. Then its group of units $R^{*}$ is $\left\{1,3, \ldots, 2^{m}-1\right\} . R^{*}$ has a subgroup $H$ of order 2: $\left\{1,2^{m}-1\right\}$. It is easy to see that $R^{*} / H$ is cyclic of order $2^{m-2}$. Define an automorphism $f$ of $R^{*}$ by $f(a)=a^{3}$. Then $f$ will be the identity on $H$, thus inducing an automorphism $\tilde{f}$ of $R^{*} / H$. Since $\left(2^{m-2}, 3\right)=1$, the elements of $R^{*} / H$ will form a single orbit under the action of $\tilde{f}$.

We can identify $R^{*}$ with the set of irreducible free $C\left[Z / 2^{m} Z\right]$-modules by choosing a fixed generator $\mu$ of $Z / 2^{m} Z$ and letting $s$ correspond to the module $M_{s}$ with $\chi_{M_{s}}(\mu)=\exp \left(\pi i s / 2^{m-1}\right)$.

Under this identification, the automorphism $f$ of $R^{*}$ will correspond to the automorphism of $M_{1}, \ldots, M_{2^{m-1}}$ induced by the action of $\psi^{3}$. Further, modding out by the subgroup $H$ corresponds to identifying conjugate pairs of representations. Consequently all the irreducible free $R\left[Z / 2^{m} Z\right]$-modules form an orbit under the action of $\psi^{3}$. This completes the proof.

Corollary 7.5. Let $G$ be a cyclic 2-group, let $V$ and $W$ be faithful irreducible $C[G]$-modules and let $\bar{W}$ be the conjugate representation of $W$. Then either $\psi^{3^{r}}(V)=W$ for some $r$ or $\psi^{3}(V)=\bar{W}$ for some $r$. 
Lemma 7.6. Suppose $G$ is generalized quaternion, dihedral, or semidihedral and suppose that $M$ and $N$ are faithful irreducible $R[G]$-modules such that $\operatorname{Dim}(M, G)=$ $\operatorname{Dim}(N, G)$. Then $\psi^{3^{r}} M=N$ for some positive integer $r$.

Proof. Since all these groups have an abelian normal subgroup $H$ of index 2, we can deduce from [24, Prop. 24] that the complex dimension of any faithful irreducible $C[G]$-module must be 2 . Thus $\operatorname{dim}_{R} M=4$ or 2 . If $\operatorname{dim}_{R}(M)=4$, then $\operatorname{End}_{R[G]}(M)=C$ or $H$. If $\operatorname{dim}_{R}(M)=2$ then $\operatorname{End}_{R[G]}(M)=R$. We consider these two cases separately.

If $\operatorname{dim}_{R} M=4$, we can deduce from [24, Theorem 16] the existence of irreducible $H$-modules $V$ and $W$ such that $M=\operatorname{ind}_{H}^{G} V$ and $N=\operatorname{ind}_{H}^{G} W$. Since $\psi^{3^{r}} V=W$ for some $r$ by the previous lemma and since $\psi^{3^{r}}$ commutes with ind ${ }_{H}^{G}$ [22, p. 70], we obtain the desired result in this case.

If $\operatorname{dim}_{R} M=2$, we will show that $\psi^{3^{r}}(M \oplus M)=N \oplus N$ and then deduce the result. We consider $M \otimes_{R} C$ and $N \otimes_{R} C$. These are irreducible $C[G]$-modules of dimension 2. Hence from [24, Theorem 16] we deduce the existence of irreducible $C[H]$-modules $V$ and $W$ such that $M \otimes_{R} C=\operatorname{ind}_{H}^{G} V$ and $N \otimes_{R} C=\operatorname{ind}_{H}^{G} W$. Observe that since $N \otimes_{R} C$ is self-conjugate, $N \otimes_{R} C=\operatorname{ind}_{H}^{G} \bar{W}$ where $\bar{W}$ is the conjugate representation to $W$. From the previous lemma then, we see that either $\psi^{3^{r}} V=W$ for some $r$, or $\psi^{3^{r} V}=\bar{W}$. Thus $\psi^{3^{r}}\left(M \otimes_{R} C\right)=N \otimes_{R} C$. We can now obtain our desired result by ignoring the complex structure (since the Adams operations commute with realification) and dividing by two. This completes the proof.

We can now deduce Theorem 7.1 from Lemmas 7.4 and 7.6.

We now turn to the proof of Theorem 7.2. This is an easy consequence of the following result of tom Dieck [7, 9.1.2], which we reformulate slightly.

THEOREM 7.7. Let $G$ be a finite group of order $g$ and let $\Gamma$ be the group of units of $Z / g Z$. Let $\Gamma$ act on $\operatorname{Irr}(G)$ by $a \cdot M=\psi^{a}(M)$ if $a \in \Gamma$ and $M$ is irreducible. Let $Z[\Gamma]$ be the integral group ring and let $I(\Gamma)$ be its augmentation ideal. Let $R O_{0}(G)$ be the additive subgroup of $R O(G)$ consisting of all elements $x$ such that $\operatorname{Dim}(x, G)=0$. Then $R O_{0}(G)=I(\Gamma) R O(G)$.

Observe that if $\operatorname{Dim}(M, G)=\operatorname{Dim}(N, G)$ then $M-N \in R O_{0}(G)$. We make the obvious generalization of our notion of "the coefficient in a $G$-module $W$ of an irreducible $G$-module $V$ " to the case in which $W$ may be virtual. Then Theorem 7.2 can be reformulated as saying that if $x \in R O(G)$ and $i(x)$ is the sum of the coefficients in $x$ of all the irreducibles in the $i$ th equivalence class $\mathcal{C}_{i}$, then $x \in R O_{0}(G)$ if and only if each $i(x)=0$.

If each $i(x)=0$, then clearly $x \in R O_{0}(G)$. It remains to prove the other implication. Observe first that the functions $i($ ?) are additive; thus if $x, y \in R O(G)$ are such that each $i(x)=0$ and each $i(y)=0$ then $i(x+y)=0$ for each $i$. Observe also that since $Z[\Gamma]$ acts on $R O(G)$ via Adams operations, we can think of an element $A \in I(\Gamma)$ as a sum $\Sigma_{j} a_{j} \psi^{k_{j}}$, where $\left(k_{j}, g\right)=1$ for each $j$ and $\Sigma_{j} a_{j}=0$. Now suppose given $z \in R O(G)$. Then $z$ can be written as $\sum_{i} z_{i}$ where all the irreducibles in the $i$ th summand $z_{i}$ are from the $i$ th equivalence class $\bigodot_{i}$. Since $\left(k_{j}, g\right)=1$ for each 
$j$, every Adams operation $\psi^{k_{j}}$ acts by a permutation on $\operatorname{Irr}(G)$, preserving the equivalence classes $\bigodot_{i}$. Hence

$$
\psi^{k_{j}}(z)=\sum_{i} \psi^{k_{j}}\left(z_{i}\right) \text { and } i\left(\psi^{k_{j}}\left(z_{i}\right)\right)=i\left(z_{i}\right) .
$$

Since $i(z)=i\left(z_{i}\right)$, we see that $i(A z)=\left(\sum_{j} a_{j}\right) \cdot i(z)$, which is zero as we noted above. Since by Theorem 7.7 any element of $R O_{0}(G)$ is a sum $\Sigma_{t} A_{t} z_{t}$ where $A_{t} \in I(\Gamma)$ and $z_{t} \in R O(G)$, Theorem 7.2 follows.

8. The Burnside ring for general universes. In this section we introduce $A(G, \mathscr{Q})$, the Burnside ring for the universe $\mathcal{Q}$, and prove some results about it which we will need in the next section. Its behaviour generally parallels that of the Burnside ring $A(G)$. However, there are a few nasty exceptions which we discuss at the end of the section.

We begin with some definitions. Let $\mathcal{Q}$ be a $G$-universe. Observe that $\operatorname{Iso}(\mathcal{Q})$ is closed under intersection, since $G_{x \oplus y}=G_{x} \cap G_{y}$. Define $\omega_{G}^{0}(\mathcal{Q})$ to be $\operatorname{colim}_{V \subset \mathcal{Q}}\left[S^{v}, S^{v}\right]_{G}$ and abbreviate this to $\omega_{G}^{0}$ when $\mathscr{Q}$ contains all irreducible $G$-modules. Since maps between the one point compactifications of complex $G$-modules are determined by their degrees on fixed point sets [7,8.4.1], it follows that the natural map $\omega_{G}^{0}(\mathcal{Q}) \rightarrow \omega_{G}^{0}$ is an injection. Let $\psi: A(G) \rightarrow \omega_{G}^{0}$ be the well-known isomorphism (e.g. [7, 8.5.1]) and define $A(G, \mathcal{Q})$ to be $\psi^{-1}\left(\omega_{G}^{0}(\mathcal{Q})\right)$. Let $I(\mathcal{Q})$ denote a subset of Iso( $(\mathcal{U})$ consisting of one group from each conjugacy class. Then the following lemma gives the additive structure of $A(G, \mathcal{Q})$.

LEMma 8.1. $A(G, \mathcal{Q})$ is the subgroup of $A(G)$ generated by $\{[G / H] \mid H \in I(\mathscr{Q})\}$.

Proof. If $G / H \subset V \subset \mathcal{Q}$, then $\psi([G / H])$ can be represented by a $G$-map $f$ : $S^{v} \rightarrow S^{v}$. Thus the subgroup generated by $\{[G / H] \mid H \in I(\mathscr{Q})\}$ is contained in $A(G, \mathscr{Q})$.

It remains to show the other containment. Let $\mathcal{C}(G)$ be a collection of conjugacy class representatives of subgroups of $G$. It is well known that the following diagram commutes, where $\phi_{H}(x)$ is the cardinality of $x^{H}, \phi=\prod_{H \in \mathcal{C}_{(G)}} \phi_{H}$, and $\gamma(f)=$ $\Pi_{H \in \mathcal{C}_{(G)}} \operatorname{deg} f^{H}$.

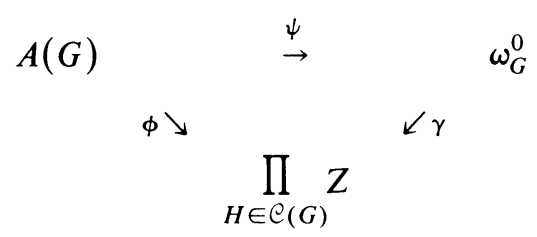

Suppose $\Sigma a_{i}\left[G / H_{i}\right] \in A(G, \mathcal{Q})$ and not all the $H_{i}$ 's lie in Iso( $\left.\mathcal{Q}\right)$. By subtracting off the cosets that are in Iso( $(\mathcal{Q})$, we obtain an element $z=\sum_{j} a_{j}\left[G / H_{j}\right]$ such that none of the $H_{j}$ 's are in Iso( $\mathcal{Q}$ ). Let $K$ be maximal among them up to conjugacy. Observe that if we denote the order of $W K$ by $|W K|$ then $\phi_{K}(z)=a_{K}|W K|$, which is not zero. However, since $z \in A(G, \mathcal{Q}), \psi(z)$ can be represented by a $G$-map $f$ : $S^{v} \rightarrow S^{v}$ for some $V \in \mathcal{Q}$. Since $K$ is not in Iso( $\left.\mathcal{Q}\right), V^{K}=V^{L}$, where $L$ is the smallest subgroup in $\operatorname{Iso}(V)$ containing $K$. Consequently $\operatorname{deg} f^{K}=\operatorname{deg} f^{L}$ and 
$\phi_{K}(z)=\phi_{L}(z)$. Since $K$ is subconjugate to none of the $H_{j}$ 's, by maximality, neither is $L$. Thus $z$ has no $L$-fixed points and $\phi_{L}(z)=0$, a contradiction. This completes the proof.

We now compute the prime ideals of $A(G, Q)$. Let $p$ denote a prime number or zero and let $q(H, p ; \mathcal{Q})=\left\{x \in A(G, \mathcal{Q}) \mid \phi_{H}(x) \equiv 0(p)\right\}$. Then this is clearly a prime ideal. We see in the following lemma that all prime ideals are of this form.

LEMMA 8.2. Let a be a prime ideal of $A(G, \mathcal{Q})$ and let $p$ be the characteristic of $A(G, \mathcal{Q}) / a$. Then there exists a unique $H \in I(\mathscr{Q})$ with $(|W H|, p)=1$ such that $a=q(H, p ;$ थ $)$.

Proof. Using Lemma 8.1, this lemma can be proven in exactly the same way as [7, 5.7.2].

REMARK 8.3. If $p \neq 0$ then $q(H, p ; \mathcal{Q})$ is a maximal ideal since $A(G, \mathcal{Q}) / q(H, p ;$ U) is a field, and $q(H, 0 ;$ Q $)$ is a minimal prime ideal since $A(G$, थ $) / q(H, 0 ;$ थ $) \cong Z$.

Let $\Phi(p)=\{H \in I(\mathcal{Q}) \mid(|W H|, p)=1\}$. We have the following splitting theorem for $A(G, \mathcal{Q})_{(p)}$-modules. The proof is analogous to that for $A(G)_{(p)}$-modules which can be found in $[17, \S 4]$.

Proposition 8.4. If $p \neq 0$ and $M$ is an $A(G, \mathcal{Q})_{(p)}$-module, then the natural map

is an isomorphism.

$$
f: M \rightarrow \bigoplus_{H \in \Phi(p)} M_{q(H, p ; \text { थ })}
$$

Recently Lewis, May, McClure, and Waner [19] have defined cohomology theories graded on the representations in a universe $\mathcal{Q}$. They show that such theories can be represented by coordinate free $G$-spectra indexed on $\mathcal{Q}$. Such theories are easily seen to give modules over $A(G, \mathcal{Q})$ in each grading. We have the following excision result for such cohomology theories.

THEOREM 8.5 (EXCISION). Let $G$ be a finite group and let $X$ be a G-CW complex. Let $h_{G}^{*}$ be $a$ थ-graded cohomology theory and let $p$ be prime to the order of $G$. Then the restriction map

$$
i^{*}: h_{G}^{*}(X)_{q(K, p ; \mathcal{Q})} \rightarrow h_{G}^{*}\left(X^{(K)}\right)_{q(K, p ; \mathcal{Q})}
$$

is an isomorphism for each $K<G$, where $X^{(K)}=G \cdot X^{K}$.

This is an immediate consequence of Propositions 8.6 and 8.7 below. The proof of 8.6 is analogous to the proof of $[\mathbf{1 7}, 4.5]$. Proposition 8.7 can easily be proven by an induction on cells.

Proposition 8.6. Suppose $K$ and $L$ are in $I(\mathcal{Q})$. Let $A(K$, थ $(K))$ be an $A(G, \mathcal{Q})$ module under the restriction map. Then $A(K, \mathcal{Q}(K))_{q(L, p ; \mathcal{Q})}$ is nonzero if and only if there is some $H<K$ with $H \in \operatorname{Iso}(\mathcal{Q})$ such that $q(H, p ; \mathcal{Q})=q(L, p ; \mathcal{Q})$.

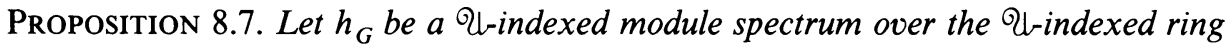
spectrum $k_{G}$. Let $X$ be a G-CW complex such that $\operatorname{Iso}(X) \subset \operatorname{Iso}(\mathcal{Q})$. Let $S=\{H \in$ Iso(थ) $\left.\mid k_{G}^{*}(G / H)=0\right\}$ and let $X^{S}=\left\{x \in X \mid G_{x} \notin S\right\}$. Then $i^{*}: h_{G}^{*}(X) \rightarrow h_{G}^{*}\left(X^{s}\right)$ is an isomorphism. 
We can now prove Theorem 8.5 .

Proof of 8.5. If $p$ is prime to the order of $G$ then $\Phi(p)=I(\mathscr{Q})$. Thus, using Lemma 8.2, Proposition 8.6 can be reformulated as follows:

$A(K, \mathscr{U}(K))_{q(L, p \text {; थ })}$ is nonzero if and only if $L$ is subconjugate to $K$.

Since any $थ$-graded cohomology theory is module-valued over $\omega_{G}^{0}(\mathcal{U})$ and since $\omega_{G}^{0}(\mathscr{U})(G / K)$ is naturally isomorphic to $A(K, \mathcal{Q}(K))$, Theorem 8.5 follows from Proposition 8.7.

We conclude this section by discussing the problems which arise when $\mathscr{Q}$ is not $G R^{\infty}$. The basic one is that Dress' characterization of when two prime ideals $q(H, p ; \mathcal{U})$ and $q(K, p ; \mathcal{U})$ are equal does not always work. In [8], Dress defined the subgroup $H_{p}$ to be the smallest normal subgroup of a given group $H$ such that the quotient $H / H_{p}$ is a $p$-group. He then proved that $q\left(H, p ; G R^{\infty}\right)=q\left(K, p ; G R^{\infty}\right)$ if and only if $H_{p}$ and $K_{p}$ are conjugate. When this characterization of prime ideals holds for $Q$ we can adapt the method of proof of $[17,4.8]$ to our situation and our excision result (Theorem 8.5) can be improved to read as follows:

THeOrem 8.8. Let $G$ be finite and let $X$ be a $G$-CW complex. Let $h_{G}^{*}$ be a U-graded cohomology theory and suppose that $K$ and $K_{p}$ are both in $\operatorname{Iso}(\mathcal{Q})$. Then the restriction map

$$
i^{*}: h_{G}^{*}(X)_{q(K, p ; \text { U })} \rightarrow h_{G}^{*}\left(X^{\left(K_{p}\right)}\right)_{q(K, p ; \text { U })}
$$

is an isomorphism.

Unfortunately, this is false for general universes even when $G$ is a cyclic group. For example, let $G=Z / 18$, let $H=Z / 9$ and let $K=Z / 3$. Since $G$ is cyclic, we can construct a universe $\mathcal{Q}$ such that $\operatorname{Iso}(\mathcal{Q})=\{G, H, K\}$. It is easy to check that $\phi_{G} \equiv \phi_{K}(2)$. Consequently $q(G, 2 ;$ थ $)=q(K, 2$; थ $)$. But $G_{2}=H$ and $K_{2}=K$ and these are evidently distinct.

The problems multiply when $H \in \Phi(p)$ and $H_{p} \notin$ Iso( $($ ).

Because of the usefulness of Theorem 8.8 we make the following definition. We will take $p=2$, but analogous definitions can be made for all sets of prime numbers.

DEFINITION 8.9. $A$ universe $Q$ is said to be excisive if

(i) for each $K \in \Phi(2), K_{2} \in \mathrm{Iso}(\mathcal{Q})$, and

(ii) Theorem 8.8 holds for any $K \in \Phi(2)$, any $G-C W X$, and any U-graded cohomology theory $h_{G}^{*}$.

Unfortunately we have found no convenient way of characterizing excisive universes.

9. The proof of Theorem C. Throughout this section $G$ will denote a group of odd order and $M$ a fixed $G$-module. All the $G$-cohomology theories used in this section will be $थ(M)$-graded, so, to simplify our notation, we will abbreviate $\mathscr{Q}=\mathscr{Q}(M)$. We will rely heavily on the techniques of J. E. McClure [21]. Since we are in a somewhat different situation, our arguments are simpler.

In view of Theorem 5.3 and the known solution to the nonequivariant vector field problem, Theorem $\mathrm{C}$ is an immediate corollary of the following theorem, which we will spend the rest of this section proving. 
Theorem 9.1. $M \xi-\mathbf{M}$ vanishes in $J O_{G}(P(k) \text {; } थ)_{(2)}$ if and only if $M^{H} \xi-\mathbf{M}^{H}$ vanishes in $J O(P(k))$ for each $H<G$.

Before proceeding to the proof, we need some terminology. Let $h$ be a multiplicative $G$-cohomology theory (थ-graded, of course), and let $E$ be a $G$-vector bundle over a space $X$ with trivial $G$-action such that $E_{x}=M$ for each $x \in X$. Given a class $\nu \in h_{G}^{M}(T E)$, we write $\nu_{x}$ for its image under the composite

$$
h_{G}^{M}(T E) \stackrel{i_{x}^{*}}{\rightarrow} h_{G}^{M}\left(T E_{x}\right) \stackrel{\iota_{x}}{\cong} h_{G}^{M}\left(S^{M}\right) \cong h_{G}^{0}\left(S^{0}\right) .
$$

We say that $\nu$ is an $h_{G}^{*}$-orientation for $E$ in dimension $M$ if each $\nu_{x}$ is a unit in $h_{G}^{0}\left(S^{0}\right)$. If $F$ is another $G$-vector bundle over $X$ such that $S\left(E_{x}\right) \sim_{G} S\left(F_{x}\right)$, we say that a fibrewise $G$-map $f: S(E) \rightarrow S(F)$ has odd $G$-degree if $f_{x}^{H}: S\left(E_{x}\right)^{H} \rightarrow S\left(F_{x}\right)^{H}$ has odd degree for each $H<G$ and $x \in X$. In the following lemma, we show that stable trivializations of $G$-sphere bundles are equivalent to orientations in stable $G$-cohomotopy.

LemMA 9.2. Let $E$ be a G-vector bundle over a G-trivial space $X$ with $E_{x}=M$ for some $M \subset$ Q and each $x \in X$.

(i) $E$ has an $\omega_{G}^{*}(?)_{(2)}$-orientation in dimension $M$ if and only if there exists a G-module $W \subset$ Q and a fibrewise G-map $f: S^{E \oplus \mathbf{W}} \rightarrow S^{\mathbf{M} \oplus \mathbf{W}}$ of odd G-degree.

(ii) Given an $H<G, E$ has an $\omega_{G}^{*}(?)_{q(H, 2 ; \mathcal{Q})}$-orientation in dimension $M$ if and only if there exists $W \subset \mathcal{Q}$ and a fibrewise G-map $f: S^{E \oplus \mathbf{W}} \rightarrow S^{\mathbf{M} \oplus \mathbf{W}}$ such that $f_{x}^{H}$ has odd degree for each $x \in X$.

Proof. We will prove (ii). The proof of (i) is similar. Suppose $\nu \in \omega_{G}^{M}(T E)_{q(H, 2 ; \text { थ })}$ is an orientation. Then, by Proposition 8.4, there is an odd integer $t$ such that $t \nu$ corresponds to a $G$-map $f: \Sigma^{W} T E \rightarrow S^{M \oplus W}$ for some $W \subset \mathcal{Q}$. Clearly $t \nu$ will still be an orientation. But if $(t \nu)_{x}$ is to be a unit, then $\left(i_{x} f\right)^{H}:\left(\Sigma^{W} T E_{x}\right)^{H} \rightarrow\left(S^{M \oplus W}\right)^{H}$ must have odd degree. Hence, if $p_{1}: S^{E \oplus \mathbf{W}} \rightarrow X$ is the projection and $p_{2}: S^{E \oplus \mathbf{W}} \rightarrow$ $\Sigma^{W} T E$ is the quotient map, then $p_{1} \times f p_{2}: S^{E \oplus \mathbf{W}} \rightarrow S^{\mathbf{M} \oplus \mathbf{W}}$ is the desired fibrewise $G$-map.

Conversely, suppose given a fibrewise $G$-map $f: S^{E \oplus \mathbf{W}} \rightarrow S^{\mathbf{M} \oplus \mathbf{W}}$ such that $W \subset U$ and $\operatorname{deg} f_{x}^{H}$ is odd for each $x \in X$. Then passage to Thom complexes, followed by projection, gives a $G$-map $\tilde{f}: \Sigma^{W} T E \rightarrow S^{M \oplus W}$. Clearly $\operatorname{deg}\left(i_{x} \tilde{f}\right)^{H}$ is also odd, so it follows that $\tilde{f}$ is an orientation.

We are now ready to prove Theorem 9.1. Clearly, the vanishing of $M \xi-\mathbf{M}$ in $J O_{G}(P(k) ; \mathscr{Q})_{(2)}$ implies the vanishing of $M^{H} \xi-\mathbf{M}^{H}$ in $J O(P(k))$ for each $H<G$. Thus it remains to prove the other half of Theorem 9.1. Since the proof is somewhat technical, we describe the general strategy before giving the details.

First, to ensure that all subgroups of $G$ act orientably, we replace $M \xi$ by $M \xi \oplus \mathbf{M}$, which we shall denote by $E$, and $\mathbf{M}$ by $\mathbf{M} \oplus \mathbf{M}$, which we shall denote by $\mathbf{N}$. Now observe that our hypotheses imply the existence of $\omega_{H}^{*}(?)_{(2)}$-orientations for $E^{H}$ in dimension $N^{H}$ for each $H<G$. Using them, we will, in stages, construct an $\omega_{G}^{*}(?)_{(2)}$ orientation for $E$ in dimension $N$. Then, using Lemma 9.2 and the equivariant Dold theorem $\bmod k[13,0.2]$, we will show that this implies the vanishing of $E-\mathbf{N}$ in 
$J O_{G}(P(k) ; \mathcal{Q})_{(2)}$. We do all this in the following four lemmas, each of which is a consequence of its predecessor.

LEMma 9.3. For each $H<G$ there exists an $\omega_{H}^{*}(?)_{q(H, 2 ; \text { Q })}$ orientation $\mu_{H}$ for $E$ in dimension $N$.

LEMmA 9.4. There is a unit $u_{H} \in A(G, \mathcal{Q})_{q(H, 2 ; \mathcal{Q})}$ such that if $\nu_{H}$ denotes $\operatorname{ind}_{H}^{G}\left(u_{H} \mu_{H}\right)$, then $\nu_{H}$ is an $\omega_{G}(?)_{q(H, 2 ; \text { Q })}$ orientation for $E$ in dimension $N$.

LEMMA 9.5. Let $\nu=\bigoplus_{H<G} \nu_{H}$, interpreted as an element of $\omega_{G}^{N}(T E)_{(2)}$ using Proposition 8.4. Then $\nu$ is an $\omega_{G}^{*}(?)_{(2)}$-orientation for $E$ in dimension $N$.

LEMMA 9.6. There is an odd integer $b$ and a fibre G-homotopy equivalence $f$ : $\left(b S^{E}\right) \rightarrow\left(b S^{\mathbf{N}}\right)$.

Clearly Theorem 9.1 is an immediate consequence of Lemma 9.6.

The proofs of these lemmas will occupy the rest of this section.

Proof of 9.3. By hypothesis, we have an $\omega_{H}^{*}(?)_{(2)}$ orientation $\tilde{\mu}_{H}$ for $E^{H}$ in dimension $N^{H}$. Clearly $\tilde{\mu}_{H}$ is also an $\omega_{H}^{*}(?)_{q(H, 2 ; \mathcal{Q})}$ orientation. We can represent $\tilde{\mu}_{H}$ by an $H$-map $f: \Sigma^{a_{H}} T E^{H} \rightarrow S^{N^{H}+a_{H}}$, where $a_{H}$ is some positive integer. Let $N_{H}$ be the complementary $H$-module to $N^{H}$ in $N$ and, for simplicity, let $a$ denote the ideal $q(H, 2 ;$ U $)$. Now consider the following sequence of $A(G, \mathcal{Q})_{a}$-module maps:

$$
\omega_{H}^{N_{H}^{H}}\left(T E^{H}\right)_{a}^{\stackrel{\Sigma^{N_{H}}}{\rightarrow}} \omega_{H}^{N}\left(T\left(E^{H} \oplus \mathbf{N}_{H}\right)\right)_{a} \stackrel{i_{1}}{\rightarrow} \omega_{H}^{N}\left(T E^{H}\right)_{a} \stackrel{i_{2}}{\leftarrow} \omega_{H}^{N}(T E)_{a} .
$$

Observe that these maps are all isomorphisms and are compatible with restriction to fibres. (The suspension map $\Sigma^{N_{H}}$ is evidently an isomorphism and $i_{1}$ and $i_{2}$ are because of Theorem 8.5.) Let $\mu_{H}=i_{2}^{-1} i_{1} \Sigma^{N_{H}}\left(\tilde{\mu}_{H}\right)$. Since $\tilde{\mu}_{H}$ restricts to an $A(H, \mathcal{Q}(H))_{a}$-module generator of $\omega_{H}^{N H}\left(T E_{x}\right)_{a}$ for each $x \in P(k), \mu_{H}$ restricts to an $A(H, \mathcal{Q}(H))_{a}$-module generator of $\omega_{H}^{N}\left(T E_{x}\right)_{a}$ for each $x \in P(k)$. Thus $\mu_{H}$ is an $\omega_{H}^{N}(?)_{a}$ orientation for $E$ in dimension $N$.

Proof of 9.4. We begin by computing $\operatorname{res}_{H}^{G}$ ind $_{H}^{G}\left(\mu_{H}\right)$. We remark that the double coset formula [7,6.1.7] is valid for Q -graded cohomology theories if the groups involved are in Iso( $($ ). (See [28].) Consequently

$$
\operatorname{res}_{H}^{G} \operatorname{ind}_{H}^{G}=\sum_{g H \in G / H} \operatorname{ind}_{H \cap g^{-1} H g}^{H} C_{g^{*}} \operatorname{res}_{g H g^{-1} \cap H}^{H},
$$

where $C_{g^{*}}$ is the transfer associated to the $G$-map $C_{g}: G / H \cap g H g^{-1} \rightarrow G / g^{-1} H g \cap H$ given by $C_{g}\left(a\left(H \cap g^{-1} H g\right)\right)=a g\left(g H g^{-1} \cap H\right)$.

Let $u_{H}$ be a unit in $A(G, \mathcal{Q})_{a}$ such that $u_{H} \mu_{H}$ can be represented by an $H$-map $f$ : $\Sigma^{W} T E \rightarrow S^{W \oplus N}$.

(Note that $W$ can be taken to be a $G$-module by suspending $f$, if necessary, by the complementary $H$-module to $W$ in the $G$-module generated by $W$ in $Q$. We can also arrange for $N_{G}(H)$ to act orientably on $S^{W^{H}}$ by replacing $W$ by $W \oplus W$.) By means of the above double coset formula, we will show that $\operatorname{deg}\left(\operatorname{res}_{H}^{G} \operatorname{ind}_{H}^{G}(f)\right)_{x}^{H}$ is odd for each $x \in P(k)$. We can then deduce Lemma 9.4 from Lemma 9.2.

First we establish that, for the purpose of computing mapping degrees, we may ignore all summands in the double coset formula which correspond to cosets $g H$ with $g \notin N_{G}(H)$. 
LEMMa 9.7. Let $K(\mathrm{~g})$ denote $\mathrm{gHg}^{-1} \cap \mathrm{H}$ and let

$$
\phi=\sum_{g H \notin W H} \operatorname{ind}_{g^{-1} K(g) g}^{H} C_{g^{*}} \operatorname{res}_{K(g)}^{H}(f) .
$$

Then $\operatorname{deg} \phi_{x}^{H}=0$ for each $x \in P(k)$.

Proof. We can represent the $g^{-1} K(g) g$-map $C_{g^{*}} \operatorname{res}_{K(g)}^{H}(f)$ by a map

$$
\tilde{f}:\left(H / g^{-1} K(g) g\right)^{+} \wedge \Sigma^{W} T E \rightarrow S^{W \oplus N} .
$$

Let $\tau: S^{V} \rightarrow S^{V} \wedge\left(H / g^{-1} K(g) g\right)^{+}$be the transfer map. Then $\operatorname{ind}_{g^{-1} K(g) g}(\tilde{f})$ corresponds to the $H$-map given by the following composite:

$$
S^{V} \wedge \Sigma^{W} T E \stackrel{\tau \wedge 1}{\rightarrow} S^{V} \wedge\left(H / g^{-1} K(g) g\right)^{+} \wedge \Sigma^{W} T E \stackrel{1 \wedge \tilde{f}}{\rightarrow} S^{V \oplus W \oplus N} .
$$

Since $g H \notin W H, g^{-1} K(g) g$ is a proper subgroup of $H$. Thus $H / g^{-1} K(g) g$ has no fixed points so $\phi_{x}$ must factor through an $H$-space with only one $H$-fixed point. Consequently deg $\phi_{x}^{H}=0$ for each $x \in P(k)$.

Thus, for the purpose of computing $\operatorname{deg}\left(\operatorname{ind}_{H}^{G}(f)\right)_{x}^{H}$, we may consider $\operatorname{res}_{H}^{G}$ ind $_{H}^{G}(f)$ to be a sum over all distinct cosets $n H \in W H$ of $H$-maps of the form

$$
\Sigma^{W} T E \stackrel{n}{\rightarrow} \Sigma^{W} T E \stackrel{f}{\rightarrow} S^{W \oplus N^{n^{-1}}} \rightarrow S^{W \oplus N} .
$$

Since we have arranged for all elements of $N_{G}(H)$ to act by degree 1 maps on $\Sigma^{W^{H}} T E^{H}$ and $S^{N^{H} \oplus W^{H}}$, it follows that $\operatorname{deg}\left(\operatorname{res}_{H}^{G} \operatorname{ind}_{H}^{G}(f)\right)_{x}^{H}=|W H| \cdot \operatorname{deg} f_{x}^{H}$ for each $x \in P(k)$. Since this is an odd number, Lemma 9.4 follows from Lemma 9.2.

Proof of 9.5. Observe that $\omega_{G}^{*}(X)_{(2)}$ is an $A(G, \mathcal{Q})_{(2)}$-module. Hence, by Proposition 8.4,

$$
\omega_{G}^{*}(X)_{(2)} \cong \bigoplus_{H \in \Phi(p)} \omega_{G}^{*}(X)_{q(H, 2 ; \mathcal{Q})} .
$$

We consider what happens when $X=T E$. Let $\nu$ be the sum of all the orientations $\nu_{H}$ constructed in Lemma 9.4. Then $\nu$ can be interpreted as an element of $\omega_{G}^{N}(T E)_{(2)}$. We will show that $\nu$ is an orientation for $E$. Let $i_{X}: T E_{X} \rightarrow T E$ be the inclusion. Since the splitting of Proposition 8.4 is natural, it follows that $i_{x}^{*}(\nu)=\Sigma_{H \in \Phi(p)} i_{x}^{*}\left(\nu_{H}\right)$. If we identify $\omega_{G}^{N} T E_{x}$ in the usual fashion with $A(G, \mathcal{Q})$, then each $i_{x}^{*}\left(\nu_{H}\right)$ corresponds to a unit in $A(G, \mathcal{U})_{q(H, 2 ; \mathcal{Q})}$. Consequently $i_{x}^{*}(\nu)$ will be a unit in $A(G, \mathcal{Q})_{(2)}$. Thus $\nu$ is an $\omega_{G}^{*}(?)_{(2)}$ orientation for $E$ in dimension $N$.

Proof of 9.6. Let $t$ be an odd integer chosen so that $t \nu$ can be represented by a $G$-map $\Sigma^{V} T E \rightarrow S^{V \oplus N}$ where $V \subset \mathcal{Q}$. Invoking Lemma 9.2, we see that this map induces a fibrewise $G$-map

$$
\gamma: S^{E \oplus \mathbf{V}} \rightarrow S^{\mathbf{N} \oplus \mathbf{V}}
$$

such that $\operatorname{deg} \gamma_{x}^{H}$ is odd for every $x \in P(k)$ and $H<G$. We will show that $\gamma$ satisfies the hypotheses of the equivariant Dold theorem $\bmod k[13,0.2]$ and will thus deduce the existence of the desired fibre $G$-homotopy equivalence.

It is enough to show that if $\alpha$ is an element of $A(G, \mathcal{Q})$ represented by $\gamma_{x}$ : $S^{E} x^{\oplus V} \rightarrow S^{N \oplus V}$, then there exists $\beta \in A(G, \mathcal{Q})$ such that $\alpha \beta=c \cdot 1$ in $A(G, \mathcal{Q})$, where $c$ is an odd number. 
Let $\phi: A(G$, थ $) \rightarrow \Pi_{H \in I(\text { थ) }} Z$ be the canonical injection. (See Lemma 8.1.) Consider $A(G, \mathcal{Q})$ as a subring of $\Pi Z$ under $\phi$. Clearly $(\Pi Z)_{(2)}$ will be integral over $A(G, \mathcal{U})_{(2)}$. Since $\alpha$ is a unit of $\Pi Z$ that lies in $A(G, \mathcal{U})_{(2)}$, its inverse, $\tilde{\beta}$, also lies in $A(G, \mathcal{Q})_{(2)}[5,5.10]$. Let $c$ be an odd number such that $c \tilde{\beta}$ lies in $A(G, \mathscr{Q})$. Then $c \tilde{\beta}$ is the desired $\beta$. This completes the proof.

10. The proof of Theorem D. Throughout this section $G$ will denote an arbitrary finite group. Recall that, for $H<G, H_{2}$ denotes the smallest normal subgroup of $H$ with 2-primary index and $\Phi(2)$ denotes the set $\{H<G \mid W H$ is odd $\}$.

The proof of Theorem D is similar to that of Theorem C. Again, we rely heavily on the methods of McClure [21]. The main part of the proof is contained in the following theorem, in which $\mathcal{Q}(M)$ need not be excisive.

THEOREM 10.1. Suppose given an $\omega_{H}^{*}(?)_{q(H, 2 ; \mathcal{Q}(M))}$ orientation for $M \xi$ in dimension $M$ for each $H \in \Phi(2)$. Then $M \xi-\mathbf{M}$ vanishes in $J O_{G}(P(k) \text {; } \mathscr{Q}(M))_{(2)}$.

Proof. We proceed as in the previous section. Observe that our hypotheses are equivalent to the conclusion of Lemma 9.3. With this input, the obvious generalizations of Lemmas 9.4, 9.5 and 9.6 to the case of finite groups follow, their proofs being exactly the same. Then, as in the case of Theorem C, Theorem 10.1 is an immediate consequence of Lemma 9.6.

Now suppose that $\mathscr{Q}(M)$ is an excisive universe (see Definition 8.9). Then Lemma 9.3 can also be generalized to the case of an arbitrary finite group.

LEMma 10.2. Suppose that $\mathscr{Q}(M)$ is an excisive universe, that $H \in \Phi(2)$, and that $M^{H_{2}} \xi-\mathbf{M}^{H_{2}}$ vanishes in $J_{H_{H} H_{2}}\left(P(k) \text {; थ }\left(M^{H_{2}}\right)\right)_{(2)}$. Then there exists an $\omega_{H}^{*}(?)_{q(H, 2 ; \mathcal{Q}(M))}$ orientation for $M \xi$ in dimension $M$.

Theorem $\mathrm{D}$ is an immediate consequence of Theorem 5.3, Theorem 10.1, and the preceding lemma.

We remark that the obstruction to generalizing this proof to all $G$-modules is the failure of excision (Theorem 8.8) in this generality. If some other method for constructing $\omega_{H}^{*}(?)_{q(H, 2 ; \mathcal{Q}(M))}$ orientations for $M \xi$ in dimension $M$ for each $H \in \Phi(2)$ were found, then Theorem D could be generalized accordingly.

11. The proof of Theorem $\mathbf{F}$. Throughout this section $G$ will denote a finite abelian group. Here we will compute the $G$-field numbers for $G$-modules $M$. These results follow easily from Theorem A and Becker's calculations [6] of the $G$-field numbers of $G$-modules on which $G$ acts freely.

Recall the decomposition of our $G$-module $M$ as a sum of submodules $M_{i}$ described above the statement of Theorem F. With the notations of that theorem, we deduce from $[6,1.1]$ the existence of $\min \left\{\alpha\left(k_{1}(M)\right), \beta\left(k_{2}(M)\right)\right\} G$-fields on each $S\left(M_{i}\right)$. Taking the joins of these $G$-fields will yield the same number on $S(M)$. Thus it suffices to show that this is an upper bound on the number of $G$-fields that may occur on $S(M)$. Equivalently, it is enough to show that the largest $k$ for which $M \xi-\mathbf{M}=0$ in $J O_{G}\left(P(k)\right.$; थ ) (or $J O_{G}(P(k))$ for that matter), is

$$
\min \left\{\alpha\left(k_{1}(M)\right), \beta\left(k_{2}(M)\right)\right\}+1 \text {. }
$$


We will first prove this for cyclic 2-groups, then prove it for all cyclic groups, and finally reduce the general case to the cyclic case.

LEMMA 11.1. Theorem $\mathrm{F}$ holds if $G$ is a cyclic 2-group.

Proof. Observe that if $G$ is a cyclic 2-group then $G$ has no type II irreducibles. Further, all the type I irreducibles must be of type $R$ and all the type III irreducibles must be of type $C$. Since all inequivalent $G$-modules with the same isotropy subgroup are permuted in a single orbit by $\psi^{3}$ (Lemma 7.4), we can conclude that $k_{1}(M)=w_{1}(M)$ and that $k_{2}(M)=w_{2}(M)$. The conclusion follows from Lemma 6.6 and the discussion preceding it.

We now treat the case in which $G$ is an arbitrary cyclic group.

LEMMA 11.2. Theorem $\mathrm{F}$ holds if $G$ is a finite cyclic group.

Proof. Let $2^{n}$ be the order of $\Sigma_{2}$, the 2-Sylow subgroup of $G$. As before, let $\Phi(2)=\{H<G \mid[G: H]$ is odd $\}$, and, given $H \in \Phi(2)$, let $H_{2}$ denote the smallest subgroup of $\mathrm{H}$ such that $\mathrm{H} / \mathrm{H}_{2}$ is a 2-group. Observe that if $M$ is a $G$-module, $k$ $G$-fields on $S(M)$ will induce $k H / H_{2}$-fields on $S\left(M^{H_{2}}\right)$. We will use the preceding lemma to show that

$$
\min _{H \in \Phi(2)}\left\{H / H_{2} \text {-field number of } M^{H_{2}}\right\} \leqslant \min \left\{\alpha\left(k_{1}(M)\right), \beta\left(k_{2}(M)\right)\right\},
$$

thus completing the proof. We treat the case $|G|$ even, the case $|G|$ odd being similar but simpler.

Observe that $H \in \Phi(2)$ if and only if $\Sigma_{2}<H$ so that $H=H_{2} \oplus \Sigma_{2}$. Further, there are only two $G$-modules of type $\mathrm{I}$, the trivial $G$-module $R$ and $L$, on which $Z / 2 Z=G / A$ acts freely, where $A$ is the unique subgroup of $G$ with index 2 . We define some subsets of $\left\{M_{1}, \ldots, M_{r+s+t}\right\}$ below. Let

$$
\begin{array}{r}
\{M(0,1), \ldots, M(0, a(H, 0))\}=\left\{M_{r+i} \mid 1 \leqslant i \leqslant s, H_{2}<J_{i} \text { and }\left|G / J_{i}\right| \text { is odd }\right\} \\
\{M(1,1), \ldots, M(1, a(H, 1))\}=\left\{M_{r+i} \mid 1 \leqslant i \leqslant s, H_{2}<J_{i},\right. \text { and the 2-Sylow } \\
\text { subgroup of } \left.G / J_{i} \text { is } Z / 2 Z\right\},
\end{array}
$$

and, for $2 \leqslant m \leqslant n$,

$$
\begin{aligned}
\{M(m, 1), \ldots, M(m, a(H, m))\}=\left\{M_{r+s+i} \mid 1 \leqslant i \leqslant t, H_{2}<K_{i},\right. \text { and the } \\
\text { 2-Sylow subgroup } \left.G / K_{i} \text { is } Z / 2^{m} Z\right\} .
\end{aligned}
$$

Let $b(R)$ and $b(L)$ denote the coefficients in $M$ of $R$ and $L$, respectively, and let $b(m, j)$ denote the number of irreducible summands in $M(m, j)$. Then

$$
M^{H_{2}}=b(R) R \oplus b(L) L \oplus \sum_{m=0}^{n} \sum_{i=1}^{a(H, m)} M(m, i) .
$$

Thus, if $M^{H_{2}}$ is thought of as an $H / H_{2}$-module, then $k_{1}\left(M^{H_{2}}\right)$ is the greatest common divisor of

$$
\{b(R), b(L), 2 b(m, i) \mid m=0 \text { or } 1,1 \leqslant i \leqslant a(H, m)\}
$$


and $k_{2}(M)$ is the greatest common divisor of

$$
\{b(m, i) \mid 2 \leqslant m \leqslant n, 1 \leqslant i \leqslant a(H, m)\} .
$$

Further, if $H, H^{\prime} \in \Phi(2)$ and $H^{\prime}<H$, then

$$
\{M(m, 1), \ldots, M(m, a(H, m))\} \subset\left\{M(m, 1), \ldots, M\left(m, a\left(H^{\prime}, m\right)\right)\right\}
$$

for each $m$. Thus, by considering chains of groups in $\Phi(2)$, it is easy to see that there must be some $H \in \Phi(2)$ such that $k_{1}\left(M^{H_{2}}\right)=k_{1}(M)$. Consequently,

$$
\min _{H \in \Phi(2)} \alpha\left(k_{1}\left(M^{H_{2}}\right)\right)=\alpha\left(k_{1}(M)\right) \text {. }
$$

A similar argument shows that

$$
\min _{H \in \Phi(2)} \beta\left(k_{2}\left(M^{H_{2}}\right)\right)=\beta\left(k_{2}(M)\right) .
$$

Hence

$$
\min _{H \in \Phi(2)}\left\{\min \left\{\alpha\left(k_{1}\left(M^{H_{2}}\right)\right), \beta\left(k_{2}\left(M^{H_{2}}\right)\right)\right\}\right\}=\min \left\{\alpha\left(k_{1}(M)\right), \beta\left(k_{2}(M)\right)\right\} .
$$

This completes the proof.

We now turn to the general situation of arbitrary finite abelian groups.

Order the set of all subgroups of $G$ having cyclic quotients by inclusion. (Note that, since $G$ may be assumed to be noncyclic, the identity group does not occur in this set.) Let $\left\{H_{1}, \ldots, H_{m}\right\}$ be the minimal elements. Clearly the $G$-field number for $M$ can be no larger than the $G / H_{i}$-field number of $M^{H_{i}}$. Thus, the proof of Theorem $\mathrm{F}$ will be complete once we show that

$$
\min _{i}\left\{G / H_{i} \text {-field number of } M^{H_{i}}\right\} \leqslant \min \left\{\alpha\left(k_{1}(M)\right), \beta\left(k_{2}(M)\right)\right\} .
$$

This is easy to see once we have the correct terminology. Analogously to the proof of Lemma 11.2, we let

$$
\begin{aligned}
& \{M(1,1), \ldots, M(1, r(i))\}=\left\{M_{j} \mid 1 \leqslant j \leqslant r, H_{i}<L_{j}\right\}, \\
& \{M(2,1), \ldots, M(2, s(i))\}=\left\{M_{r+j} \mid 1 \leqslant j \leqslant r, H_{i}<J_{j}\right\},
\end{aligned}
$$

and

$$
\{M(3,1), \ldots, M(3, t(i))\}=\left\{M_{r+s+j} \mid 1 \leqslant j \leqslant t, H_{i}<K_{j}\right\} .
$$

As before let $b(i, j)$ be the number of irreducible submodules of $M(i, j)$. Then it is easy to see that

$$
M^{H_{i}}=\sum_{m=1}^{3} \sum_{j} M(m, j) .
$$

Further, since the $M(1, j)$ 's are still type I and so on, it follows that $k_{1}\left(M^{H_{i}}\right)$ and $k_{2}\left(M^{H_{i}}\right)$ are the greatest common divisors, respectively, of

$$
\{b(1,1), \ldots, b(1, r(i)), 2 b(2,1), \ldots, 2 b(2, s(i))\}
$$

and

$$
\{b(3,1), \ldots, b(3, t(i))\}
$$


We deduce from Lemma 11.2 that $\min \left\{\alpha\left(k_{1}\left(M^{H_{i}}\right)\right), \beta\left(k_{2}\left(M^{H_{i}}\right)\right)\right\}$ is the $G / H_{i}$-field number for $M^{H_{i}}$. Since $H_{1}, \ldots, H_{m}$ is a complete list of minimal elements, every $M_{q}$ must occur as an $M(m, j)$ for some $H_{i}$. Thus,

$$
\min _{i}\left\{\alpha\left(k_{1}\left(M^{H_{i}}\right)\right)\right\}=\alpha\left(k_{1}(M)\right) \text { and } \min _{i}\left\{\beta\left(k_{2}\left(M^{H_{i}}\right)\right)\right\}=\beta\left(k_{2}(M)\right),
$$

so that

$$
\min _{i}\left\{\min \left\{\alpha\left(k_{1}\left(M^{H_{i}}\right)\right), \beta\left(k_{2}\left(M^{H_{i}}\right)\right)\right\}\right\}=\min \left\{\alpha\left(k_{1}(M)\right), \beta\left(k_{2}(M)\right)\right\} .
$$

This completes the proof of Theorem F.

12. Construction of $G$-fields. In this section we explicitly construct $G$-fields on $S(M)$. Recall that a $G$-field $\nu$ on $S(M)$ can be interpreted as a $G$-map $\nu: S(M) \rightarrow$ $S(M)$ such that $\nu(x) \perp x$ for each $x \in S(M)$. Such maps can easily be constructed using Clifford algebras.

The standard Clifford algebra $C_{k}$ can be thought of as the free $R$-algebra generated by the elements $e_{1}, \ldots, e_{k}$ subject to the relations $e_{i}^{2}=1$ and $e_{i} e_{j}+e_{j} e_{i}=0$ if $i \neq j$. Say that a $G$-module $M$ has a $C_{k} G$-structure (or is a $C_{k} G$-module) if it is a $C_{k}$-module and the $G$-action commutes with the $C_{k}$-action.

The proof of the following result is exactly the same as in the nonequivariant case. See $[15,11(2.4)]$.

Proposition 12.1. A $C_{n} G$-module structure on $M$ gives rise to $n$ G-fields on $S(M)$.

We now investigate which $G$-modules admit a $C_{n} G$-structure. We begin by recalling some useful facts about Clifford algebras from [4].

Let $F(t)$ denote the algebra of $t \times t$ matrices over $F$, where $F$ is $R, C$ or $H$. Then each $C_{n}$ is a sum of such matrix algebras, as indicated in the following table.

\begin{tabular}{l|l}
\multicolumn{1}{c|}{$n$} & $C_{n}$ \\
\hline $8 d$ & $R\left(2^{4 d}\right)$ \\
$8 d+1$ & $C\left(2^{4 d}\right)$ \\
$8 d+2$ & $H\left(2^{4 d}\right)$ \\
$8 d+3$ & $H\left(2^{4 d}\right) \oplus H\left(2^{4 d}\right)$
\end{tabular}

\begin{tabular}{c|l}
$n$ & $C_{n}$ \\
\hline $8 d+4$ & $H\left(2^{4 d+1}\right)$ \\
$8 d+5$ & $C\left(2^{4 d+2}\right)$ \\
$8 d+6$ & $R\left(2^{4 d+3}\right)$ \\
$8 d+7$ & $R\left(2^{4 d+3}\right) \oplus R\left(2^{4 d+3}\right)$
\end{tabular}

The algebra $F(t)$ has a unique simple module of dimension $t$ over $F$. It can be thought of as the column vector space $F^{t}$ on which $F(t)$ acts by left multiplication. Further, $\operatorname{End}_{F(t)}\left(F^{t}\right) \cong F$, and these endomorphisms correspond to scalar multiplication on the right of $F^{t}$.

We now consider the algebra of $F(t)$ endomorphisms of $\oplus_{1}^{k} F^{t}$. We can identify End $_{F(t)}\left[\oplus_{1}^{k} F^{t}\right]$ with $F(m)$ in the following manner. Let $\iota_{j}$ denote the inclusion of the $j$ th factor of $\bigoplus_{1}^{k} F^{t}$ and let $\pi_{j}$ denote the projection onto the $j$ th factor. Given an endomorphism $\alpha$ of $\bigoplus_{1}^{k} F^{t}$, let $A \in F(m)$ be the matrix whose $(i, j)$ th entry is the scalar corresponding to the $F(t)$ map $\left(\pi_{i} \alpha \iota_{j}\right): F^{t} \rightarrow F^{t}$. Then $\alpha$ can be induced by multiplying the row vector space $\bigoplus_{1}^{k} F^{t}$ on the right by $A \otimes I_{(t)}$, the matrix tensor product of $A$ with $I(t)$, the unit in $F(t)$. 
Suppose now that $M$ admits a $C_{n} G$-structure. Then the $G$-action induces a group homomorphism $\tilde{\rho}: G \rightarrow$ Aut $_{C_{n}}(M)$, and thus a map $\bar{\rho}: G \rightarrow \operatorname{End}_{C_{n}}(M)$. Since we have computed End $_{C_{n}}(M)$ to be $F(k) \otimes I(t)$ (or $[F(a) \otimes I(t)] \oplus[F(b) \otimes I(t)]$ if $n \equiv 3$ or 7 (8)), $\bar{\rho}$ corresponds to a map $\rho: G \rightarrow F(k) \otimes I(t)$. Observe that $\operatorname{dim} M=$ $t \cdot k \cdot \operatorname{dim}_{R} F$ (or $t(a+b) \operatorname{dim}_{R} F$ if $n \equiv 3$ or $7(8)$ ). Further, if $G$ acts orthogonally on $M$, then the image of $\rho$ must be contained in the appropriate unitary group: $O(k) \otimes I(t), U(k) \otimes I(t)$ or $S p(k) \otimes I(t)$. Using the preceding table, we can describe the group of orthogonal $C_{n}$-automorphisms of $C_{n}$-modules in the following table:

$$
\begin{array}{ll}
C_{8 d} & O(k) \otimes I\left(2^{4 d}\right) \\
C_{8 d+1} & U(k) \otimes I\left(2^{4 d}\right) \\
C_{8 d+2} & S p(k) \otimes I\left(2^{4 d}\right) \\
C_{8 d+3} & S p(k) \otimes I\left(2^{4 d}\right) \text { or } S p(a) \otimes I\left(2^{4 d}\right) \oplus S p(b) \otimes I\left(2^{4 d}\right) \\
C_{8 d+4} & S p(k) \otimes I\left(2^{4 d+1}\right) \\
C_{8 d+5} & U(k) \otimes I\left(2^{4 d+2}\right) \\
C_{8 d+6} & O(k) \otimes I\left(2^{4 d+3}\right) \\
C_{8 d+7} & O(k) \otimes I\left(2^{4 d+3}\right) \text { or } O(a) \otimes I\left(2^{4 d+3}\right) \oplus O(b) \otimes I\left(2^{4 d+3}\right)
\end{array}
$$

Using this table we can compute the largest $n$ for which $M$ admits a $C_{n} G$-structure. Observe that if the $G$-action on $M$ is induced by a homomorphism from $G$ to $O(m) \otimes I(t)$, then $M$ is isomorphic to a $t$-fold direct sum of some submodule $N$. In view of this, we say that a positive integer $t$ divides $M$ if $M$ can be written as a $t$-fold direct sum of some submodule. Let $k$ be the largest power of 2 dividing $M$, so that $M=2^{k} N$ for some submodule $N$. Write $N$ as a sum $N_{1} \oplus N_{2} \oplus N_{3}$, where $N_{1}$ is of type $R, N_{2}$ of type $C$, and $N_{3}$ of type $H$. Let $k_{1}, k_{2}$, and $k_{3}$, respectively, be the largest powers of 2 dividing $N_{1}, N_{2}$, and $N_{3}$. (We take $k_{i}=\infty$ if $N_{i}=0$.) Note that one of the $k_{i}$ 's must be zero or else $k$ will not be maximal. Thus, using the preceding table, and considering all possible values for the $k_{i}$ 's, we can list in the following table the largest $n$ for which various $G$-modules $M$ admit $C_{n} G$-structures:

$k_{1}=0$
$k_{1}=1$ or $k_{1} \geqslant 2, k_{2}=0$
$k_{1} \geqslant 2, k_{2} \geqslant 1$$\quad \begin{array}{llll}8 d+1 & 8 d+2 & 8 d+4 & 8 d+8 \\ 8 d+2 & 8 d+4 & 8 d+6 & 8 d+8 \\ 8 d+4 & 8 d+5 & 8 d+6 & 8 d+8\end{array}$

\section{BIBLIOGRAPHY}

1. J. F. Adams, Vector fields on spheres, Ann. of Math. (2) 75 (1962), 603-632.

2. On the groups $J(X)$. II, Topology 3 (1965), 137-171.

3. M. F. Atiyah, Thom complexes, Proc. London. Math. Soc. (3) 11 (1961), 291-310.

4. M. F. Atiyah, R. Bott and A. Shapiro, Clifford modules, Topology 3 (supplement 1) (1964), 3-38.

5. M. F. Atiyah and I. G. MacDonald, Introduction to commutative algebra, Addison-Wesley, Reading, Mass., 1969. 
6. J. C. Becker, The span of spherical space forms, Amer. J. Math. 94 (1972), 991-1026.

7. T. tom Dieck, Transformation groups and representation theory, Lecture Notes in Math., vol. 766, Springer-Verlag, Berlin and New York, 1979.

8. A. Dress, $A$ characterization of solvable groups, Math. Z. 110 (1969), 213-217.

9. B. Eckmann, Beweis des Satzes von Hurwitz-Radon, Comment. Math. Helv. 15 (1942), 358-366.

10. D. Gorenstein, Finite groups, Harper \& Row, New York, 1968.

11. A. Haefliger and M. W. Hirsch, Immersions in the stable range, Ann. of Math. (2) 78 (1963), 223-255

12. H. Hauschild, Aquivariante Homotopie. I, Arch. Math. 29 (1977), 158-165.

13. H. Hauschild and S. Waner, Equivariant Dold theorem $\bmod k$ and the Adams conjecture, Illinois $\mathrm{J}$. Math. (to appear).

14. H. Hopf, Ein topologischer Beitrang zur rellen Algebra, Comment. Math. Helv. 13 (1940), 219-230.

15. D. Husemoller, Fibre bundles, Springer-Verlag, Berlin and New York, 1966.

16. I. M. James, Cross sections of Stiefel manifolds, Proc. London Math. Soc. (3) 8 (1958), 536-47.

17. C. Kosniowski, Equivariant cohomology and stable cohomotopy, Math. Ann. 210 (1974), 83-104.

18. L. G. Lewis, J. P. May, J. E. McClure and M. Steinberger, Equivariant stable homotopy theory, Lecture Notes in Math., Springer-Verlag, Berlin and New York (to appear).

19. L. G. Lewis, J. P. May, J. E. McClure and S. Waner, Equivariant cohomology theory (to appear)

20. T. Matumoto, On G-CW complexes and a theorem of J. H. C. Whitehead, J. Fac. Sci. Univ. Tokyo Sect. IA Math. 18 (1971/72), 363-374.

21. J. E. McClure, On the groups $J O_{G}(X)$. I, Math. Z. (to appear).

22. D. Quillen, The Adams conjecture, Topology 10 (1971), 67-80.

23. G. B. Segal, Equivariant K-theory, Inst. Hautes Études Sci. Publ. Math. 34 (1968), 129-151.

24. J.-P. Serre, Linear representations of finite groups, Springer-Verlag, Berlin and New York, 1977.

25. D. Sjerve, Vector bundles over orbit manifolds, Trans. Amer. Math. Soc. 138 (1969), 97-106.

26. S. Waner, $G-C W(V)$ complexes (preprint; to appear as part of [19]).

27. , Equivariant homotopy theory and Milnor's theorem, Trans. Amer. Math. Soc. 258 (1980),

$351-368$.

28. __ Equivariant fibrations and transfer, Trans. Amer. Math. Soc. 258 (1980), 369-384.

29. L. M. Woodward, Vector fields on spheres and a generalization, Quart. J. Math. Oxford Ser. (2) 24 (1973), 357-366.

30. M. F. Atiyah and D. O. Tall, Group representations, $\lambda$-rings and the J-homomorphism, Topology 8 (1969), 253-297.

Department of Mathematics, University of Chicago, 5734 University Avenue, Chicago, ILLINOIS 60637 